Ortwin Renn/Thomas Webler

\title{
Der kooperative Diskurs: Grundkonzeption und Fallbeispiel
}

\begin{abstract}
Complex modern societies require new ways of political conflict solution, especially concerning environmental conflicts. We distinguish six forms of conflict solution, including those of mediated bargaining and cooperative discourse. If one opts for cooperative discourse, further orientation according to criteria such as fairness, competence, legitimation and efficiency seems to be important. Three procedural steps within cooperative discourse-i.e. establishing relevant value attitudes, expert-hearings, evaluation of options by citizen panels-are sketched and critically discussed, making use of experience from recent discoursive siting panels in Switzerland and Southern Germany. With the exception of a legitimatory deficiency (which may be improved) cooperative discourse and citizen participation in environmental decision-making turn out to be most promising.
\end{abstract}

\section{Problemaufriß: Notwendigkeit diskursiver Konfliktaustragung}

In der modernen Soziologie ist es üblich, die komplexen Strukturen der Gesellschaft in einzelne Subsysteme zu untergliedern (Parsons 1967; Luhmann 1984). Dabei werden vor allem die gesellschaftlichen Subsysteme ,Wirtschaft', ,Politik', ,Kultur' und ,Soziales' hervorgehoben, die jeweils unterschiedliche Strukturen und Funktionsabläufe im Rahmen der dort ablaufenden Interaktionen aufweisen. Während für Wirtschaft und Politik erfolgsorientierte Formen der Interaktion vorherrschen, also auf die strategische Durchsetzung von Interessen ausgerichtet sind, bestimmen in stärkerem Maße verständigungsorientierte Strukturen die sozialen und kulturellen Prozesse der Interaktion (Habermas 1971, 101ff.; 1981, Bd. I, 141ff., 369ff.). Erfolgsorientierte, strategische Interaktion ist strukturell davon geprägt, daß Akteure ihre Ziele durchsetzen wollen, indem sie Interaktion als Mittel zum Ausgleich der jeweils eigenen Interessen nutzen. Demgemäß erfolgt Überzeugung im Rahmen des ökonomischen Handlungsumfeldes durch ,Bargaining', d.h. durch Anreize, Drohungen, Versprechungen, Kompensationen oder Hinweise auf Abwanderungsoptionen, oder im Rahmen des politischen Handlungsfeldes durch ,Autorität', d.h. durch 
Machtansprüche, Verweis auf verbindliche Rechte und Pflichten sowie durch Androhung von Sanktionen. ${ }^{1}$ Verständigungsorientiertes, auf Argumentation aufbauende Interaktion ist dagegen an die Bedingung geknüpft, daß die Interaktionspartner gleichberechtigt und ohne äußeren Zwang ihre Interessen und Werte offenlegen und durch Austausch von Argumenten eine gemeinsame Lösung anstreben (Apel 1990, 37ff.; Habermas 1991, 68ff.). Die Gültigkeit der Argumente ist dabei an logische Widerspruchsfreiheit und Unparteilichkeit gebunden (Saretzki 1996, 23). Im kulturellen Rahmen beziehen sich Gültigkeitsansprüche auf die Wahrung der jeweiligen kollektiven Identität (einschließlich der damit einhergehenden Regeln), im sozialen System auf Plausibilität und unmittelbare Evidenz.

Solche groben Klassifizierungen sind immer problematisch, weil sie allzu pauschal die gesellschaftlichen Subsysteme über einen Kamm scheren, sie verweisen jedoch auf einige interessante Strukturbrüche, durch die moderne Gesellschaften in Legitimationsprobleme geraten. Die heute allseits beklagte Politikverdrossenheit wie auch der umgekehrte Vorwurf der Politik an die Anspruchsmentalität der Bürger mögen ihre Ursache darin haben, daß die erforderliche Balance zwischen verständigungsorientiertem und erfolgsorientiertem kommunikativem Handeln gestört ist (Renn 1996, 162). Auf der einen Seite erhebt das Subsystem ,Wirtschaft' zunehmend den Anspruch, auch das soziale und politische Handeln mit der ihr eigenen Logik der Marktes zu kolonisieren, andererseits wird die Lebenswelt im sozialen Bereich zunehmend von politischen Einflüssen durchsetzt und das Alltagsleben durch professionalisierte Eliten fremdbestimmt (Habermas 1981, Bd. II, 293; Prätorius 1990, 65; Biesecker 1996, 12ff.). Die verbleibenden sozialen Verständigungsprozesse verkümmern häufig zu Ritualen der kollektiven Orientierung, Solidarität äußert sich nur noch in massenwirksamen Aktionen organisierter Interessen, und soziales Vertrauen bildet sich allenfalls noch im primären Kreis von Verwandten und Freunden, während die Außenwelt als zunehmend anonym und bedrohlich empfunden wird (vgl. Habermas 1981, Bd. II, 275ff.).

Wie kann unter diesen Umständen gesamtgesellschaftliche Steuerungspolitik aufrecht erhalten werden? Wie kann das politische System mit dem Konflikt zwischen der faktischen Vielfalt von akteursspezifischen Rationalitäten und der notwendigen integrativen Steuerungsrationalität des Gemeinwesens umgehen? Gibt es Verfahren, die eine plurale Eingabe von Werten, Interessen

1 Elster unterscheidet in diesem Zusammenhang, arguing' und ,bargaining'. Im ersten Falle ergibt sich die Übereinkunft auf der Basis von Gültigkeitsansprüche der Argumente, im zweiten Fall auf der Basis von Versprechungen, Kompensationen oder Androhungen von Abwanderung. Nach dieser Kategorisierung sind die kulturellen und sozialen Formen der Kommunikation bei aller Verschiedenheit eher auf ,arguing' aufgebaut, in der Terminologie von Habermas also verständigungsorientiert, die politischen und ökonomischen Formen der Kommunikation eher auf ,bargaining' bezogen, in der Terminologie von Habermas also strategieorientiert. Siehe: Elster 1989; vgl. auch dazu Saretzki 1996, 20ff. Siehe auch Abschnitt 3 in diesem Beitrag. 
und Vorstellungen bei gleichzeitiger Ausgabe von allgemein-verpflichtenden Verhaltensvorschriften oder Planungsvorgaben erlauben?

Im folgenden stellen wir zunächst eine Systematik vor, die unterschiedliche Vorgehensweisen beleuchtet, um gesamtgesellschaftliche Steuerungsprozesse im Bereich der Umweltpolitik zu organisieren und zu legitimieren. Um eine sinnvolle Auswahl der Steuerungsprozesse vornehmen zu können, leiten wir im dritten Abschnitt aus der Systematik vier Kriterien zur Bewertung von Entscheidungsverfahren für kollektiv verbindliches Handeln ab. Dann wenden wir uns dem Modell des kooperativen Diskurses zu, das wir als Instrument zur Konfliktaustragung und akteursbezogenen Planung auf der Basis des diskursiven Planungsansatzes entwickelt haben. Schließlich schildern wir ein Fallbeispiel aus dem Kanton Aargau in der Schweiz, bevor wir einige allgemeine Schlußfolgerungen ziehen.

\section{Grundtypen der Konfliktlösung in demokratischen Gesellschaften}

Welche Formen der Konfliktaustragung wären im Prinzip geeignet, bei umweltrelevanten Fragen eine demokratisch legitimierte und sachlich gerechtfertigte Lösung zu finden? Welche Typen von Konfliktlösungsverfahren gibt es, um Auseinandersetzungen zwischen der Bevölkerung von Standortgemeinden und Planern, zwischen Behörden und Bürgern, zwischen verschiedenen Lagern von Experten zu schlichten? Die heute praktizierten Verfahren der Entscheidungsfindung können als Manifestationen von vier verschiedenen Grundmodellen in Analogie zu den Grundsystemen der Gesellschaft oder aber als Kombinationen von mindestens zwei Modellen verstanden werden (siehe Bild 1 auf der nächsten Seite).

Die marktbestimmten Modelle umfassen finanzielle Anreizsysteme, property-rights-Übertragung und Kompensationsausgleiche, die expertokratischen Modelle alle auf Expertenwissen beruhenden Entscheidungsverfahren (etwa Delphi u.a.), die regulativen Modelle legale Verfahren der Beschlußfassung und -überprüfung sowie die diskursiven Modelle alle Formen verständigungsund konsensorientierter Entscheidungsfindung. Darüber hinaus gibt es Kombinationen zwischen den Grundmodellen. Mediation, Bargaining und Verhandlungslösungen können zum Beispiel als Bindeglieder zwischen Marktmodell und Diskurs interpretiert werden, während etwa die dezisionistische Arbeitsteilung zwischen Experten und politischen Repräsentanten eine Kombination von wissensorientierten und regulativen Steuerungselementen darstellt. Im folgenden sind die wesentlichen Modelle politischer Entscheidungsfindung näher beschrieben (vgl. dazu die drei Politikberatungsmodelle in Habermas 1968 und die Steuerungsmodelle von Macht, Geld und Wissen bei Willke 1995). 
Bild 1: Strukturen kollektiv bindender Entscheidungsprozesse

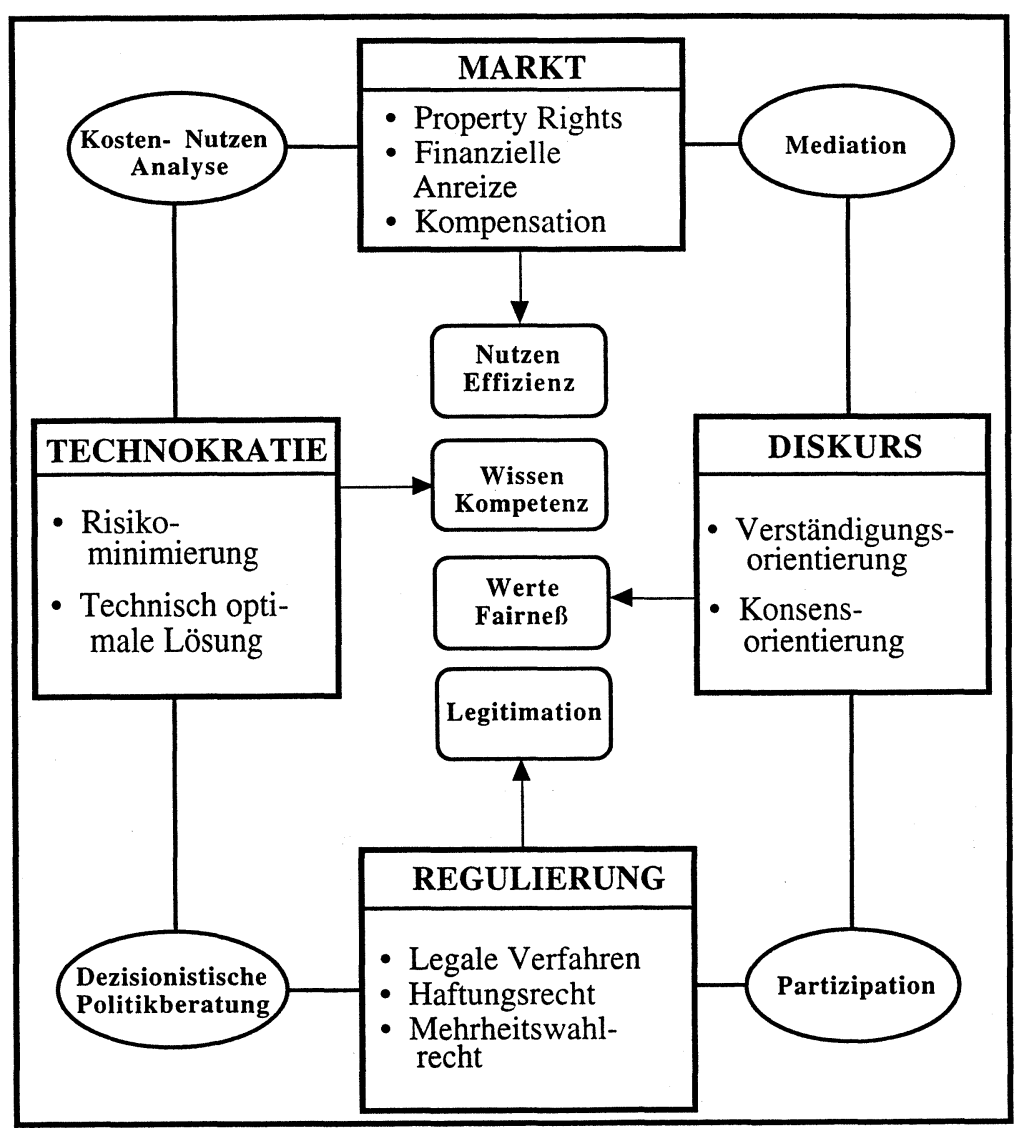

a) Expertokratie: In diesem Grundmodell kommen ausschließlich Experten zum Zuge. Diese Art von technokratischer Problemlösung mag in einzelnen Fällen von der Sache her gerechtfertigt sein, sie ist jedoch nicht demokratisch (Rosenbaum 1978; DeSario/Langton 1987; Shrader-Frechette 1990). Denn jede politische Entscheidung basiert nicht nur auf dem Wissen um die möglichen Konsequenzen der Entscheidungsoptionen, sondern auch auf Präferenzen über die Wünschbarkeit dieser Auswirkungen. Experten sind aber nicht mehr als jede anderen Staatsbürger legitimiert, solche Präferenzen zu äußern. Umweltprobleme sind auch Fragen von Interessen, Werten und Gefühlen, alles legitime Elemente eines problemadäquaten Entscheidungsverfahrens, die nicht unter den Tisch vermeintlicher Sachzwänge fallen dürfen (Scarcinelli 1990, 48f.). Somit ist Sachwissen notwendig, aber nicht hinreichend. Trotz dieser Bedenken ist in der Realität diese Form der Entscheidungsfindung weiterhin beliebt, weil sie den Entscheidungsvorgang erheblich beschleunigt und den Po- 
litikern die Chance bietet, bei Fehlurteilen oder bei unpopulären Maßnahmen die Verantwortung auf die Experten zu schieben (Jasanoff 1982; Renn 1995, 149).

b) Dezisionistische Arbeitsteilung: In diesem Mischmodell zwischen Expertokratie und staatlicher Regulation sind die Experten für das Sachwissen zuständig, die politisch legitimierten Entscheidungsträger nehmen dann aufgrund dieses Wissens und ihrer Präferenzen die Entscheidungen vor (Bruder 1980, 30f.; Cupps 1977). Dahinter steht die Auffassung, daß Wissen und Interessen völlig voneinander getrennt werden können. Eine solche Trennung bereitet jedoch konzeptionelle und praktische Probleme. Erstens muß sich das Sachwissen immer auf die Dimensionen beziehen, die für die politischen Entscheider zur wissensorientierten Ausbildung der eigenen Präferenzen von zentraler Bedeutung sind. Zweitens gibt es immer denkbare oder sogar wahrscheinliche Konsequenzen, für die es keine oder eine nur unzureichende Wissensbasis gibt. Drittens ist das Sachwissen nicht unabhängig von wertenden Grundannahmen, gleichzeitig sind die wertenden Präferenzen nicht unabhängig von den damit assoziierten Wissensgrundlagen, die häufig mit den infrage stehenden Optionen nicht einmal in direkter Verbindung stehen. Viertens zeigt dieses Modell auch keine Möglichkeit auf, wie Entscheider mit Expertendissens umgehen sollen. Darüber hinaus ist es gerade das Kennzeichen von Umweltkonflikten, daß sich die Präferenzen der Entscheider (Verwaltung oder Politik) nicht mehr mit den Präferenzen vieler betroffener Bürger decken. Die oben aufgezeigten strukturellen Probleme kann das dezisionistische Modell nicht auflösen (von Schomberg 1992; Renn 1995).

c) Substitution von politischen Entscheidungen durch den Markt: Das Marktmodell geht von der Prämisse aus, daß auch kollektive Güter bei entsprechender Strukturierung der politischen Rahmenbedingungen (etwa durch die Schaffung neuer Eigentumsrechte) in das Kalkül individueller Nutzenentscheide eingebracht werden können. Dahinter steht die Auffassung, es sei eine vordringliche Aufgabe der Politik, dafür Sorge zu tragen, daß durch geeignete Rahmenbedingungen die durch den Konflikt offengelegten externen Kosten internalisiert werden (Suhr 1990, 128ff.; Akademie der Wissenschaften zu Berlin 1992, 443ff.). Maßnahmen zur Internalisierung sind notwendig, weil bei Umweltgütern der Markt als Lenkungsinstrument meistens versagt.

Wenn Umweltbelastungen auf einen überschaubaren Kreis von Betroffenen einzugrenzen sind, können direkte Kompensationen als Ausgleich für die Belastungen ausgehandelt werden (O'Hare 1977; O'Hare 1990; Kunreuther 1995). Im Idealfall ist die Ausgleichszahlung identisch mit dem monetär bewerteten Nutzenentgang durch die Umweltbelastung. In einer Reihe von Fallbeispielen ist es in der Tat gelungen, durch Kompensationen Umweltkonflikte zur Zufriedenheit aller Beteiligten zu lösen (vgl. die Beispiele in Bacow/Wheeler 1984, 344ff.; Holznagel 1986, 356f.). Verfahren, die auf Verhandlungen über 
Kompensation beruhen oder die Auktionen bzw. Lotterien einsetzen, um kosteneffizient und fair Umweltbelastungen zu verteilen, sind zwar vielfach theoretisch beschrieben (O'Hare 1990; Kunreuther/Portney 1991; Sullivan 1992 ) und auch praktisch z.T. ausprobiert worden (Himmelberger u.a. 1991). Sie sind aber dann gescheitert, wenn sie nicht zusätzlich diskursive Elemente enthielten, mit denen eine grundsätzliche Zustimmungsbereitschaft zu den Umweltbelastungen oder Risiken ausgelotet werden konnte (vgl. Frey/Oberholzer 1996). Das von einer Reihe Umweltökonomen verfaßte ,Credo' der Standortfindung betont ausdrücklich die Notwendigkeit eines fairen Aushandlungsprozesses, bevor es zu Verhandlungen über Kompensationen kommen kann (Consensus 1990; vgl. auch Kunreuther u.a. 1993). In der Regel wird Kompensation erst dann als Ausgleichsstrategie ernsthaft in Erwägung gezogen, wenn die Akzeptabilität der jeweiligen Anlage in den Augen der Betroffenen nachgewiesen ist.

d) Konfliktlösung durch (neo)korporatistische Verhandlungsstrategie: Diese Form der Konfliktlösung ist in Europa, vor allem in Großbritannien, Deutschland und der Schweiz, vorherrschend, wird aber auch in den USA unter dem Namen ,Negotiated Rule Making' angewandt (von Alemann/Heintze 1979; Wehrli-Schindler 1987; Fiorino 1990; Kelman 1992). Ziel dieser Strategie ist es, die großen gesellschaftlichen Gruppen an der Entscheidungsfindung zu beteiligen, um die Werte und Interessen dieser Gruppen bei der Eingabe der Präferenzen in die Entscheidungen zu berücksichtigen. Um strategisches Verhalten der Teilnehmer gegenüber der Außenwelt zu vermeiden, finden diese Verhandlungen meist hinter verschlossenen Türen statt. Solche korporatistischen Verhandlungsstrategien sind dann relativ effizient, wenn eine Notlage vorliegt und sich die Gruppen im Prinzip einig sind, daß gehandelt werden muß. Ist ein solcher Druck allerdings nicht vorhanden, dann liegt es meist im Interesse mindestens eines der Teilnehmer, den Prozeß so lange in Gang zu halten und Ergebnisse zu verzögern, bis der zunehmende öffentliche Druck eine Entscheidung erzwingt. Korporatistische Lösungen haben daher drei entscheidende Nachteile (Hadden 1995, 250f.): Sie schließen erstens alle die Gruppen aus, die sich nicht an die Spielregeln der nichtöffentlichen Verhandlungen halten wollen oder können, weil sie etwa sonst ihre Klienten verlieren würden (Beispiel: Umweltinitiativen); sie reflektieren zweitens nur in geringem Maße die Interessen und Werte der Betroffenen und sie führen meist zu einem Legitimationsdefizit der getroffenen Entscheidung, weil die Öffentlichkeit an dem Entscheidungsprozeß nicht hat teilhaben können (mangelnde Transparenz). Die Wahrnehmung von Intransparenz und vermuteter ,Kungelei' macht solche Umweltentscheide anfällig für öffentliche Kritik und mangelnde Akzeptanz (Renn u.a. 1995b, 346f.).

e) Konfliktlösung durch direkte Verhandlungen (Mediation): Verhandlungen zwischen den Konfliktparteien sind seit je her wichtige Instrumente der 
Konfliktaustragung. Vor allem im Bereich der Tarifauseinandersetzungen sind direkte Aushandlungsprozesse zwischen Arbeitgebern und Arbeitnehmern mit und ohne Schlichter Gang und Gäbe. In jüngster Zeit sind solche Aushandlungsprozesse auch im Bereich der Umweltpolitik eingesetzt worden. Sie werden häufig unter dem Begriff der Mediation geführt (Folberg/Taylor 1984; Hoffmann-Riem/Schmidt-Assmann 1990; Holznagel 1990; Gaßner u.a. 1992; Wiedemann 1994). Zunehmend kommen solche Verfahren auch in Deutschland zum Tragen. ${ }^{2}$ Im deutschen Rechtssystem ist der Einsatz von Mediationsverfahren aber umstritten, da rechtlich festgesetzte Rahmenbedingungen, vor allem die Nicht-Kompensierbarkeit bestimmter Tauschbeziehungen, kein Verhandlungsgegenstand sein dürfen (Brohm 1990, 257).

Bei direkten Aushandlungsprozessen sitzen sich die in einem Konflikt beteiligten Parteien als gleichberechtigte Partner an einen ,runden' Tisch gegenüber und suchen unter der Anleitung eines neutralen Schlichters eine für alle akzeptable Lösung des Konflikts. Der Schlichter hat dabei eine katalysatorische Funktion: Er ist nicht Partei, sondern versucht, den Einigungsprozeß voranzutreiben (O'Hare u.a. 1983, 154ff.; Susskind/Cruishank 1987). Gegenüber dem korporatistischen Modell zeichnet sich die direkte Verhandlungslösung dadurch aus, daß auch lokale und spontane Organisationen an der Schlichtung teilnehmen können. Ebenso können die Teilnehmer an solchen Verhandlungen ein öffentliches Forum schaffen, das den Entscheidungsprozeß den Nichtbeteiligten transparent macht. Eine auf Verhandlungen aufgebaute Lösung kommt aber nur dann zustande, wenn die am Konflikt beteiligten Parteien keine besseren Aussichten auf die Durchsetzung ihrer Interessen mit Hilfe der Konfrontation wahrnehmen oder wenn die Schnittmenge der für alle akzeptablen Problemlösungen größer Null ist (Renn/Oppermann 1995, 264f.). Darüber hinaus bedeutet die Einbindung von organisierten Interessen eine Ausgrenzung aller der Betroffenen, die sich nicht oder noch nicht organisierten Gruppen angeschlossen haben (Rushewski 1984).

f) Kooperative Verfahren unter Beteiligung der Betroffenen: Kooperative Verfahren zur Entscheidungsfindung sind darauf ausgerichtet, einen Diskurs unter den Betroffenen herbeizuführen, der auf der einen Seite sicherstellt, daß eine kompetente Problemerfassung und Problemlösung erfolgt, auf der anderen Seite aber jedem potentiell Betroffenen die gleiche Chance einräumt, seine Werte und Interessen in den Entscheidungsprozeß einzubinden (Striegnitz

2 So begleitete und evaluierte beispielsweise das Wissenschaftszentrum in Berlin ein Schlichtungsverfahren zur Konzepterstellung eines Abfallwirtschaftsplans im Kreis Neuss. Frank Claus und Peter Wiedemann haben eine Fülle von Fallbeispielen zur Mediation in einen Sammelband aus dem Jahre 1994 aufgenommen und dort kommentiert. Die Erfolge dieser Mediationsversuche sind sehr unterschiedlich zu beurteilen, je nach dem, welche Erfolgskriterien man anlegt. Einige Verfahren haben zu einem einvernehmlichen Abschluß geführt, andere sind dagegen vorzeitig beendet worden. Vgl. Fietkau 1994; Holznagel 1990; Claus/Wiedemann 1994; Gaßner u.a. 1992. 
1990, 51f.; Zilleßen 1993, 21). Um beiden Zielen gerecht zu werden, bedarf es einer Strukturierung des Verständigungsprozesses, durch den die Einbindung des notwendigen Sachwissens, die Berücksichtigung der geltenden Normen und Gesetze, die Integration von sachlichen wie emotionalen Aussagen sowie eine faire Repräsentanz der sozialen Werte und Interessen sichergestellt werden (Dryzek 1990, 46ff.).

Unsere Erfahrungen mit solchen Diskursen beziehen sich auf Umweltprobleme und Möglichkeiten ihrer Lösung. Umweltprobleme sind vor allem deshalb für kooperative Verfahren geeignet, weil sie kollektiv verbindliche Regelungen erfordern, die weder aus der Rationalität der Experten abzuleiten, noch aus der Anwendung politischer Routinen zu rechtfertigen sind (Zilleßen 1993, 23ff.; Hill 1995, 74). Bei politischen Entscheidungen über Umweltfragen spielen Probleme, wie wahrgenommene Unsicherheiten, Verteilungsungerechtigkeiten, mangelnde Transparenz des Entscheidungsverfahrens, Dissens unter Fachleuten und Perzeptionsdifferenzen zwischen Betroffenen und Entscheidern, eine zentrale Rolle.

Die kooperative Strategie verspricht eine Reihe von Vorteilen bei der Behandlung von Umweltkonflikten (und darüber hinaus). Kollektiv bindende Entscheidungen in der Umweltpolitik sind auf eine politische Festlegung von Zielen und Mitteln angewiesen. Diese Festlegung der normativen Voraussetzungen gerät zunehmend in die Legitimationsfalle traditioneller Umweltpolitik, sei sie dezisionistischer oder korporatistischer Natur (Suhr 1990, 115ff.; Wiedemann/Claus 1994, 228). Dadurch kommt es zu einer Paralyse der Politik, weil einerseits potentiell umweltschädigende Anlagen in den Strudel der Akzeptanzverweigerung geraten, andererseits die an deren Stelle notwendigen Verhaltensänderungen zu einer ökologisch besser vertretbaren Lebensform ausbleiben, da der einzelne in der Illusion der Marginalität des eigenen Verhaltens gefangen ist (zum Problem der Krise regulativer Steuerungspolitik vgl. Mayntz 1987, 89ff.; Beck 1986, 323ff.; Scarcinelli 1990, 40ff.; Willke 1995, 179ff.). Somit landet man folgerichtig beim Müllberg, der weiter anwächst, obwohl ihn niemand wachsen sehen will, während die zur Entsorgung der anwachsenden Müllberge notwendigen Technologien ihrer Risiken wegen auf Ablehnung stoßen. Diese Diskrepanz verfestigt die Immobilität der Umweltpolitik und fördert eine Verschiebung der Problemlösung (oft verbunden mit einer Verschlimmerung der Problemlage). Paralyse trägt auch maßgeblich zu der hinlänglich beklagten Politikverdrossenheit bei. Denn die Bürger erwarten zu Recht von der Politik Entscheidungsfähigkeit. Aber erst die Möglichkeit, in einem Diskurs der Betroffenen die Konsequenzen kollektiven Verhaltens zu verdeutlichen und zu thematisieren, trägt dazu bei, die Wahrnehmung von Marginalität des eigenen Verhaltens zu überwinden und gerechte Ausgleiche für auftretende Verteilungskonflikte zu entwickeln (Wissenschaftlicher Beirat 1996, 39). 
Auch wenn das Potential diskursiver Verfahren viele der heute zutage tretenden Steuerungsprobleme in der Umweltpolitik zu lösen verspricht, so sind damit auch eine Reihe von Problemen und möglichen Nachteilen verbunden. Zum einen ist die Frage nach der Auswahl der Teilnehmer an solchen Diskursen zu klären. Zum zweiten ist bei diskursiven Verfahren die Einbindung des notwendigen Sachwissens oft problematisch. Einigung auf der Basis von gemeinsamen Vorurteilen, Illusionen oder Ausblendung von unerwünschten Nebenfolgen wird auf Dauer niemanden zufrieden stellen. Zum dritten können Diskurse als raffinierte Methode der Akzeptanzbeschaffung mißbraucht werden, wenn der Spielraum für das Mandat so eng ausgelegt ist, daß vorbestimmte Entscheidungen nur noch im nachhinein eine scheindemokratische Legitimation erhalten sollen (Führ 1990). Umgekehrt liegt die Gefahr nahe, daß Themen des Diskurses einen so weiten Spielraum umfassen, daß sie keine praktische Relevanz mehr für politische Entscheidungen aufweisen, sondern nur noch Debattierzirkel über wünschenswerte Zustände der Welt abgeben (Döbert 1992).

Stellt man die Vor- und Nachteile diskursiver Verfahren gegenüber, so scheinen Diskurse aus unserer Sicht zumindest als Bestandteile einer kompetenten und fairen Umweltpolitik sinnvoll und auch notwendig zu sein, um die Steuerungsprobleme moderner Gesellschaften konstruktiv zu überwinden (Hill 1995, 73). Allerdings erfordern die mit Diskursen verbundenen Probleme eine klare Strukturierung des dialogischen Prozesses nach internen Gesprächsregeln und externen Gestaltungskriterien.

\section{Anforderungen an eine diskursive Konfliktaustragung und Entscheidungsfindung}

Die wichtigsten Anforderungen für eine diskursive Lösung von Umweltkonflikten oder eine planerische Gestaltung von Umweltaufgaben sind mit vier Schlüsselbegriffen verbunden, wie sie bereits in Bild 1 dargestellt wurden: Fairneß, Kompetenz, Legitimation und Effizienz (Renn u.a. 1995b; vgl. ein ähnliches Kategoriensystem bei Quinn/Rohrbaugh 1981; Vlek/Cvetkovich 1989, 308f., sowie Linder/Vatter 1996).

Der Begriff der Fairneß ist eng an das Konzept der Beteiligung der betroffenen Bürger an Entscheidungen angebunden. Er läßt sich in externe und interne Fairneß untergliedern. Bei der externen Fairneß geht es um die angemessene Repräsentanz aller Betroffenen in einem Diskurs. Die Teilnehmer in einem Diskurs können durch politische Legitimation (etwa Wahlen), durch von den Betroffenen explizit legitimierte Gruppen (etwa Gewerkschaften oder Umweltgruppen) oder durch formale Verfahren der Chancengleichheit (etwa Auswahl nach dem Zufallsverfahren) bestimmt werden. Aus Sicht der Chan- 
cengleichheit sind Auswahlverfahren, die auf einer Zufallsstichprobe aus der Gesamtheit der betroffenen Bürger beruhen, allen anderen Verfahren vorzuziehen, weil dann jeder der betroffenen Bürger die gleiche Chance hat, am Prozeß der Entscheidungsfindung teilzuhaben (Dienel 1989, 134). Eine solche Auswahl nach dem Zufallsverfahren setzt aber voraus, daß die Zahl der Betroffenen eingrenzbar ist, diese betroffenen Bürger ungefähr in gleichem Ausmaß durch die Entscheidungsoptionen tangiert sind und ein genügend hohes Motivationspotential zur Teilnahme vorliegt. Sind diese Voraussetzungen nicht gegeben, dann können entweder Teilnehmer nach dem Freiwilligkeitsprinzip (jeder ist eingeladen) oder nach einem nachvollziehbaren Repräsentationsschlüssel ausgewählt werden.

Neben der externen Fairneß der Repräsentanz aller betroffenen Personen bedeutet Fairneß auch Chancengleichheit innerhalb des Diskurses. Damit ist gemeint, daß alle Teilnehmer im Diskurs gleiche Rechte und Pflichten haben. Ihnen muß es möglich sein, eigene Vorstellungen zu entwickeln, Begründungen für ihre Aussagen einzubringen und die von anderen Teilnehmern eingebrachten Geltungsanspräche zu kritisieren. Interne Fairneß setzt die Einhaltung von Gesprächs- und Konfliktlösungsregeln voraus. In einem Diskurs sollten diese Regeln vorab besprochen und möglichst konsensual verabschiedet werden (Webler 1995, 62f.).

Eine faire Repräsentation aller Parteien sowie ein faire Gesprächsführung bedeutet aber noch lange nicht, daß auch die notwendige Sachkompetenz vorhanden ist, d.h. daß das Wissen um die Folgen und Nebenfolgen von unterschiedlichen Handlungsoptionen auch wirklich bei den Verhandlungen adäquat berücksichtigt wird. Der Begriff der Kompetenz wird im Alltag häufig mit Sachwissen in Anlehnung an die Zweck-Mittel Rationalität gleichgesetzt. Dabei wird Kompetenz danach beurteilt, ob sich die vorhergesagten Konsequenzen der ausgewählten Optionen auch wirklich in der Realität einstellen. Diese Auslegung ist aber wenig hilfreich, da der ex-post Zustand noch nicht eingetreten ist, also die Beurteilungsgrundlage fehlt. Im Grundsatz geht es auch nicht um objektives Wissen, sondern um die Minimierung von subjektiver Unzufriedenheit, die daraus entsteht, daß man bei der Entscheidungsfindung im Prinzip vorhersehbare Folgen und Nebenfolgen unbeachtet gelassen oder falsch wahrgenommen hat (im englischen Sprachraum treffend als 'minimization of post-decisional regret' bezeichnet). Hätten die Teilnehmer eines Diskurses Erkenntnisse über mögliche Folgen und Nebenfolgen zum Zeitpunkt der Entscheidungsfindung verfügbar gehabt, dann wären sie auf der Basis dieses Wissens möglicherweise zu einer anderen Optionenauswahl gekommen. ${ }^{3}$ Aus

3 Hier geht es nur um das Wissen, das zum Zeitpunkt der Entscheidung verfügbar gewesen wäre und dessen Kenntnis oder Beachtung eine Präferenzverschiebung hätte auslösen können. Davon zu unterscheiden ist das Problem der Unsicherheit von Folgen, das eine eindeutige Regel zur Auswahl von Optionen auch bei gegebenen Präferenzen nicht zuläßt. Ob jemand eine Vorliebe für risiko-aversives oder risiko-freudiges Verhalten zeigt, läßt sich zwar 
diesem Grunde erscheint es uns notwendig, sachliche Kompetenz an der Leistung des Verfahrens zu messen, die im Dialog gemachten Aussagen nach dem Stand des jeweiligen Wissens intersubjektiv nachvollziehbar beurteilen und selektieren zu können (Webler 1995, 58f.). Jeder Diskurs muß so strukturiert sein, daß er Möglichkeiten zur Überprüfung von Aussagen bereitstellt und er bei der Beurteilung dieser Aussagen gemeinsam vereinbarte Regeln der intersubjektiven Nachprüfbarkeit konsequent umsetzt.

Gerade der letzte Punkt weist auf eine weitere Dimension der Kompetenz hin. Die Teilnehmer müssen die Fähigkeit mitbringen oder darin eingewiesen werden, ihre Vorstellungen, Aussagen und Kritikpunkte so zu formulieren und an andere zu kommunizieren, daß eine Verständigung über die innere Logik der Argumente und deren Begründung erfolgen kann. ${ }^{4}$ Somit umfaßt Kompetenz auch die Fähigkeit, Argumente über Normen, Werte und gefühlsmäßige Einschätzungen formulieren und austauschen zu können (Habermas 1992, 260f.). Darüber hinaus muß eine auf Argumenten beruhende Auseinandersetzung mit Geltungsansprüchen erfolgen, bei der Normen der Wahrheitsfindung eingehalten und empirisches Folgewissen berücksichtigt werden. Die Theorie des kommunikativen Handelns von Habermas bietet bei allen kritischen Einwänden gegen ihre impliziten Universalisierungsansprüche (siehe dazu die Kritik bei Wellmer 1992) einen auch für den pragmatischen Vollzug realer Diskurse brauchbaren Orientierungsrahmen, der den Organisatoren und Moderatoren von Diskursen hilft, gemeinsame Regeln der Beweisführung zu vereinbaren und die Gültigkeit von Geltungsansprüchen zu überprüfen (ausführlicher dazu Webler 1995).

Drittes Stichwort: Legitimation. Legitimation bedeutet in unserem Kontext, daß die im Diskurs gefundenen Lösungen auch für Außenstehende nachvollziehbar sind und als kompetent und fair angesehen werden, so daß sie durch die Kraft der Argumente und durch den Einbezug aller relevanter Werte und Interessen Verbindlichkeit beanspruchen können (Dahl 1989, 108ff.). Es gibt kein Beteiligungsverfahren, bei dem alle betroffenen Bürger an der Entscheidung insgesamt teilnehmen können. Vielmehr sind zwei Voraussetzungen notwendig: Die demokratisch legitimierten Entscheidungsträger müssen ihre Bereitschaft kundtun, die im Diskurs gefundenen Empfehlungen offen und wohlwollend zu prüfen, die Diskursveranstalter müssen sicherstellen, daß die Fairneß bei der Auswahl der Teilnehmer am Diskurs gesichert und die Kom-

subjektiv begründen, aber nicht intersubjektiv verbindlich entscheiden. An dieser Stelle ist häufig mit einem Konsens über den Dissens zu rechnen, wie er später noch ausführlicher beschrieben wird.

4 Greifenstein u.a. unterschieden zwischen partizipativer, funktionaler und politischer Kompetenz. Partizipative Kompetenz ist bei ihnen ein Systemmerkmal, das partizipative Verfahren erst erlaubt. Funktionale und politische Kompetenz sind ähnlich definiert wie in diesem Artikel Sachkompetenz und kommunikative Kompetenz; vgl. Greifenstein u.a. 1990, $22 \mathrm{f}$. 
petenz der Teilnehmer gegenüber Außenstehenden nachgewiesen ist. In der Praxis ist dies oft schwierig zu bewerkstelligen, da die Arbeitsatmosphäre in den Diskursen Außenstehenden nur schwer vermittelbar ist und da die in einem gut strukturierten Diskurs geschaffene Verständigungsbereitschaft unter den Teilnehmern von externen Beobachtern mit großer Skepsis betrachtet wird (Gaßner u.a. 1992, 65ff.).

Vor jeder Beteiligungsmaßnahme ist daher die Einbindung der Ergebnisse in den politischen Entscheidungsprozeß zu klären. Die Politiker geben zwar keine formale Entscheidungsmacht ab, faktisch sind sie aber vor und während der Beteiligungsmaßnahme an die Offenhaltung mehrerer Problemlösungen gebunden (Hoffmann-Riem 1990, 40). Im Anschluß an die Beteiligungsmaßnahme sind sie zumindest angehalten, die Ergebnisse zu kommentieren und eventuelle Abweichungen von dem Ergebnis eingehend zu begründen. Sie müssen das Ergebnis nicht unbedingt übernehmen, aber ohne eine überzeugende Begründung abweichender politischer Entscheidungen wird das Verfahren unglaubwürdig. ${ }^{5}$ Partizipation kann nur funktionieren, wenn den Beteiligten zugetraut wird, Dinge zu bewerten und rationale Empfehlungen auszusprechen. Die vielfach beklagte Politikverdrossenheit ist oft nur die Kehrseite des Mangels an Zutrauen in die Rationalität und Gemeinwohlorientierung der Bürger durch die Politiker.

Letztes Stichwort: Effizienz. In einer Welt mit knappen Ressourcen sind auch die Mittel für Diskurse nicht unbegrenzt verfügbar. Die Ergebnisse diskursiver Prozesse müssen im angemessenen Verhältnis zum Aufwand an Zeit und Kosten stehen (Quinn/Rohrbaugh 1983, 368f.; Linder/Vatter 1996). Effizienz umfaßt noch eine zweite Dimension. Die Teilnehmer erwarten von den Diskurs-Organisatoren, daß die Verhandlungsführung effektiv und zielgerichtet erfolgt (Gaßner u.a. 1992, 63). Langatmige Grundsatzerklärungen, Übungen in rhetorischen Floskeln, Fensterreden und endlose Diskussionen nerven jeden Diskursteilnehmer und führen zum Diskursabbruch oder zu Frustrationen. Aus unserer Sicht wird man dem Ziel der effizienten Verhandlungsführung am besten dadurch gerecht, daß man eine entscheidungsanalytische Vorgehensweise wählt, in der man sukzessiv die Schritte: Erfassung von Wertorientierungen, Ableitung von Kriterien, Optionengestaltung, Gewichtungen der Kriterien, Bewertung der Entscheidungsoptionen nach den gewichteten Krite-

5 Helmut Willke sieht in der mangelnden Durchsetzungskraft einer konsensorientierten Entscheidung den entscheidenden Nachteil verständigungsorientierter Steuerung. Er schreibt: „Eine funktionierende soziale Ordnung braucht Einrichtungen, welche im Ernstfall ein Mitglied dazu zwingen können, den vereinbarten Beitrag zur Herstellung und Sicherung der Kollektivgüter zu leisten. Gegen Opportunismus, Trittbrettfahren oder Vertragsbruch ist kein anderes Kraut gewachsen. Auch nicht in der Form des Diskurses." (Willke 1995, 161) Legitimation bedeutet aber aus unserer Sicht, daß die Ergebnisse des Diskurses als Legitimationsgrundlage für politisch legales Entscheiden und Durchsetzen dienen kann und sollte. Diskurs kann hoheitliches Handeln nicht ersetzten, aber anreichern. 
rien und Modifikationen von Optionen ausführt. ${ }^{6}$ Diese Vorgehensweise stellt auf der einen Seite sicher, daß zielgerichtet an der Gestaltung und Bewertung von Optionen gearbeitet wird, hilft auf der anderen Seite aber auch, die Regeln der Einlösung von Geltungsansprüchen einzuhalten und Transparenz über auftretende Zielkonflikte herzustellen.

Diskursive Prozesse zur Entscheidungsvorbereitung in der Politik sind daran zu messen, wie sie die vier Kriterien der Fairneß, Kompetenz, Legitimation und Effizienz einhalten können. Da jedes dieser vier Kriterien einem Steuerungsprinzip primär zuzuordnen ist (Fairneß dem Diskurs, Kompetenz der Expertokratie, Legitimation dem politisch-rechtlichen System und Effizienz dem Markt), ist also der Erfolg eines Diskurses davon abhängig, wie es ihm gelingt, auf der einen Seite alternative Steuerungsprinzipien intern zu integrieren (etwa effiziente Verhandlungsführung) und auf der anderen die Anschlußfähigkeit an die externen Steuerungsmechanismen herzustellen (etwa Einbindung in politisch legale Entscheidungsabläufe).

\section{Das Modell des kooperativen Diskurses}

Wie können diskursive Verständigungsprozesse in die Praxis umgesetzt werden? Welche Form des Diskurses entspricht am besten den Kriterien der Fairneß, Kompetenz, Legitimation und Effizienz? Ein einziges Verfahren, das diese beiden Fragen befriedigend beantwortet, ist weder in Sicht noch theoretisch zu erwarten. Aus diesem Grunde ist es sinnvoll, mehrere dialogische Verfahren miteinander zu koppeln. Dazu gibt es eine ganze Reihe von Vorschlägen (vgl. die umfangreiche Materialsammlung in Renn u.a. 1995a; Renn/Oppermann 1995; Gaßner u.a. 1992; Burns/Überhorst 1988).

Aus der Erfahrung der normengenerierenden Kraft von Diskursen haben wir ein eigenes Modell entwickelt und ihm den Namen ,Kooperativen Diskurs gegeben (Renn u.a. 1991; Renn/Webler 1994; Renn 1996). Der kooperative Diskurs beruht auf einer akteursbezogenen Organisation des Planungsablaufs in drei Schritten: einen praktischen und evaluativen Diskurs zur Normenfindung und Wertstrukturierung, der Klärung von kognitiven Konflikten durch die Methode des Experten-Delphis und der Abwägung von Handlungsoptionen durch Bürgerforen (Renn u.a. 1993). Dieses Modell ist in seinen Grundzügen in zahlreichen Beispielen in der Abfallplanung, in der Umwelt- und Raumplanung sowie in Fragen der Energiepolitik erprobt worden (Renn u.a. 1985; 1989; 1991; 1996; Webler 1994; Akademie für Technikfolgenabschätzung 1994; $1995 ; 1996)$.

Das Modell folgt einem Aufbau in drei Phasen oder Schritten, wobei die

6 Im Rahmen dieses Aufsatzes wollen wir die entscheidungsanalytische Vorgehensweise, die wir bei unseren Diskursen in der Regel durchlaufen, nicht weiter erklären. Ausführliche Informationen darüber geben die Ausführungen in: Renn u.a. 1985; 1991; 1993 
Vor- und Nachteile der Verfahrensbestandteile im positiven Sinn miteinander verknüpft werden. Das Grundmodell beruht auf der sequentiellen Verknüpfung von Normenbildung bzw. Wertstrukturierung, Evidenz-Ermittlung und Abwägung von Handlungsoptionen. Diese drei Aufgaben werden vorrangig von den Akteuren vorgenommen, von denen wir annehmen, daß sie für diese Aufgabenstellung besonders geeignet seien. Die Verknüpfung dieser drei Ebenen geschieht in den folgenden drei Schritten:

(1) Alle beteiligten Parteien werden gebeten, ihre normativen Grundlagen (Prinzipien, Werte, Kriterien) für die Beurteilung unterschiedlicher Handlungsoptionen (etwa Einführung einer neuen Technologie; Modifikation vor Einführung; Ablehnung einer neuen Technologie) offenzulegen. Dies geschieht in Interviews zwischen den Diskurs-Organisatoren und den Repräsentanten der jeweiligen Parteien. Als methodisches Werkzeug dient dabei die WertbaumAnalyse, ein in den USA entwickeltes interaktives Verfahren zur Bewußtmachung und Strukturierung von Werten und Attributen (Keeney u.a. 1984; 1987). Alle Parteien haben das Recht, ihren Wertbaum solange zu modifizieren, bis sie mit dem Produkt einverstanden sind. Die Wertbäume aller Parteien werden dann additiv zu einem logischen Gesamtbaum verschmolzen, wobei alle nicht-redundanten Eingaben übernommen und in eine hierarchische Struktur überführt werden (Renn u.a. 1993). Im Idealfall wird der gemeinsame Wertbaum in einem diskursiven Prozeß der gegenseitigen Abstimmung zwischen den Vertretern der beteiligten Gruppen zusammengestellt. Als oberstes Prüfkriterium gilt bei normativen Aussagen, die im Sinne von K.O.-Kriterien den Gültigkeitsrahmen für die relative Gewichtung der Einzelwerte abstecken, die Frage nach der Universalisierbarkeit, wobei Vorteile für einzelne dann erlaubt sind, wenn andere dadurch nicht über Gebühr Schaden erleiden müssen und dem Ergebnis zustimmen können. Bei evaluativen Fragen gilt es, die Legitimität pluraler Lebensstile zu wahren und die Bedingungen zu thematisieren, die eine wertgerechte Lebensweise für alle Beteiligten erlauben, ohne daß damit andere in ihren Lebensstilen beschnitten werden. Der Gesamtwertebaum spiegelt folglich die normativen Grundlagen und die Wertdimensionen aller beteiligten Parteien wider. Die Einbeziehung aller relevanten Werte in einen logisch kohärenten Bezugsrahmen hilft, potentielle Konflikte über die Angemessenheit von Werten und Berurteilungskriterien zu entschärfen und allen Parteien das Gefühl zu vermitteln, daß ihre Bedenken in den Entscheidungsprozeß eingebunden werden.

(2) Die Wertdimensionen werden in einem zweiten Schritt durch ein Forschungsteam, das möglichst von allen Parteien als neutral angesehen wird, in Indikatoren transformiert. Dies ist der erste Schritt zur Lösung von Konflikten über Sachverhalte. Diese Indikatoren sind Meßanweisungen, um die möglichen Folgen einer jeden Handlungsoption zu bestimmen (Evidenz-Nachweise). Da viele der Folgen nicht physisch meßbar sind und manche auch wissen- 
schaftlich umstritten sein mögen, ist es nicht möglich, einen einzigen Wert für jeden Indikator anzugeben (vgl. von Schomberg 1992). Für den Diskurs ist es entscheidend, die Spannweite wissenschaftlich legitimer Abschätzungen so genau wie möglich zu bestimmen. Dazu haben wir eine Modifikation des klassischen Delphi-Verfahrens entwickelt, bei dem Gruppen von Experten gemeinsam Abschätzungen vornehmen und Diskrepanzen innerhalb der Gruppen in direkter Konfrontation ausdiskutieren (Webler u.a. 1991). Dieses Verfahren zur kognitiven Konsensfindung bezeichnen wir als ,Gruppen-Delphi'. Der Prozeß des Gruppen-Delphis steht unter dem Zeichen der Einigung oder Nichteinigung über kognitive Aussagen zu einem Problem. Er wird streng nach methodischen Regeln geführt und hat zum Ziel, scheinbare Dissense zu identifizieren und diese in Konsense aufzulösen sowie echte Dissense auf gemeinsam akzeptierte Begründungslogiken zurückzuführen und damit einen Konsens über den Dissens zu schaffen.

(3) Hat man die Wertdimensionen bestimmt und die Folgen der jeweiligen Handlungsoptionen im Sinne von Konsensen und begründeten Dissensen abgeschätzt, folgt der schwierige Prozeß der Abwägung. Es geht also um die Wahl einer Option aus vielen möglichen Handlungsoptionen, wobei diese Entscheidung auf der Basis der zu erwartenden Folgen, der persönlichen oder kollektiven Präferenzen über diese Folgen und der diskursiven Auflösung von Zielkonflikten erfolgen sollte. Bei der Komplexität solcher Abwägungen kommt es entscheidend auf die Einhaltung der wesentlichen Strukturmerkmale von verständigungsorientierten Diskursen an. Denn evaluative und normative Zielkonflikte können nicht allein durch Rekurs auf die möglichen Folgen von Optionen gelöst werden, sondern schließen die subjektiven Wünschbarkeiten dieser Folgen im Hinblick auf soziale Normen, Werte und Lebensstile ein. Hier versagen sowohl Sachwissen als Lösungsinstanz wie auch ,Bargaining' als Tauschbörse (vgl. Creighton 1983; Saretzki 1996).

Aus diesem Grund halten wir auch die in der amerikanischen Literatur vorherrschenden spieltheoretischen Ansätze zur Erklärung und Gestaltung von Verhandlungsprozessen für unzureichend (siehe Renn/Webler 1994). Die spieltheoretische Sichtweise von Diskursen als Verhandlungen beruht auf der Voraussetzung, daß die Parteien ihre Positionen zugunsten einer Interessenperspektive aufgeben. Positionen sind in diesem Rahmen nicht vermittlungsfähig, sondern nur noch Interessen (Raiffa 1982; Bacow/Wheeler 1984, 344ff.). Die Übersetzung von Positionen in Interessen ist sicherlich ein wichtiger Schritt in Richtung Konsensbildung, er reicht aber nicht aus. Die spieltheoretische Perspektive verkennt die vorrangige Leistung des Diskurses, Leitlinien einer sozialen Rationalität zu entwickeln, die über das Interesse der einzelnen Parteien hinausgehen. Ein Diskurs kann nämlich ein Instrument der Normengenerierung sein (vgl. Etzioni 1993; Chambers 1992). Erst in dem Zusammenspiel aller Interessen, Werte und Vorstellungen können neue gemeinsame Orientie- 
rungen entstehen, welche die Probleme der individuellen Rationalität zugunsten einer neuen Sichtweise von sozialer Rationalität transzendieren. Häufig einigen sich die Beteiligten auf gemeinsame neue kommunale Werte, deren Anerkennung und Geltungskraft eine Neuformulierung und ein Überdenken der eigenen Interessenlage bedingt. Eine solche Diskursleistung ist natürlich nur dort zu erwarten, wo sich die Teilnehmer aktiv für die Erarbeitung gemeinsamer Normen und Werte einsetzen und die Diskursstruktur eine Diskussion über Geltungsansprüche von normativen Aussagen zuläßt. Gerade dies geschieht aber in den spieltheoretisch aufgebauten Diskursen des Interessenausgleichs so gut wie nie. ${ }^{7}$

Konflikte über Wünschbarkeit von Handlungen lassen sich daher nur dann in fairer und kompetenter Form auflösen, wenn die betroffenen Gruppen sich in einem verständigungsorientierten Diskurs einigen. Als Teilnehmer solcher Diskurse könnten z.B. Vertreter organisierter Interessengruppen dienen. Häufig aber sind diese Parteien in ihren Meinungen weitgehend polarisiert und nicht mehr verständigungsbereit, ihnen mangelt es an den beiden wichtigen Voraussetzungen für das Gelingen eines Diskurses: Lernbereitschaft und guter Wille. Gleichzeitig repräsentieren sie nur in eingeschränktem Maß die betroffene Bevölkerung. Aus diesem Grund hat P. Dienel vorgeschlagen, die Bevölkerung als ,Schöffen' zu gewinnen und es einigen, nach dem Zufallsverfahren ausgesuchten Bürgern zu überlassen, stellvertretend für alle diese Abwägung vorzunehmen (Dienel 1978; Dienel 1989; Dienel/Renn 1995). ${ }^{8}$ Dieses Verfahren setzt voraus, daß die am Konflikt beteiligten organisierten Interessen einer solchen Lösung zustimmen oder sie zumindest tolerieren. Dies wird um so eher geschehen, je mehr die Parteien selber keine Chance mehr wahrnehmen, den Konflikt aus eigenen Kräften zu überwinden, und je eher sie davon überzeugt sind, daß sie ihren Standpunkt dem Schiedsgericht überzeugend nahebringen können. Alle Parteien sind daher eingeladen, als Zeugen auszusagen und ihre Empfehlungen vorzutragen. Die ausgesuchten Teilnehmer haben ausreichend Zeit, die Profile der jeweiligen Handlungsoptionen zu studieren, Experten zu befragen, Zeugen anzuhören, Besichtigungen vorzunehmen und sich eingehend $\mathrm{zu}$ beraten. Am Ende stellen sie eine Handlungsempfehlung aus, die sie ein-

7 „Ein praktischer Diskurs“, so die amerikanische Diskurstheoretikerin Simone Chambers, ,ist nicht einem Problem rationaler Wahl (,rational choice') gleichzusetzen. Vielmehr handelt es sich um einen Prozeß kollektiver Interpretation und Re-interpretation und demzufolge auch um die Möglichkeit der Neubildung und Umbildung von Einstellungen und Interessen der Teilnehmer. Im praktischen Diskurs als realem Gesprächsprozeß gelangen wirkliche Menschen zur Einsicht, daß bestimmte Prinzipien gerecht, anerkennungswürdig und daher bindend für sie sind." (Chambers 1992, 172)

8 Parallel zu den Arbeiten von Peter Dienel zur Planungszelle hat auch N. Crosby ein Verfahren eines Schiedsgerichtes (Citizen Juries) entwickelt, das ebenfalls auf der Idee von Laiengutachtern, die nach dem Losverfahren ausgewählt werden, beruht; vgl. Crosby u.a. 1986. Laien als Planer werden in den USA in verschiedenen Kontexten eingesetzt: als Beispiel vgl. Kathlene/Martin 1991. 
gehend in einem Bürgergutachten begründen müssen. Dafür erhalten sie eine Vergütung. Diesem Verfahren hat P. Dienel den etwas problematischen Namen ,Planungszelle' gegeben. Wir bevorzugen dagegen den Namen ,Bürgerforum‘ Bürgerforen haben sich auf kommunaler Ebene wie auf regionaler Ebene bereits bewährt und wurden erstmals für einen nationalen Konflikt zu Beginn der 80iger Jahre eingesetzt (Renn u.a. 1985; Dienel/Garbe 1985).

Bild 2: Das Drei-Stufen-Modell der Partizipation

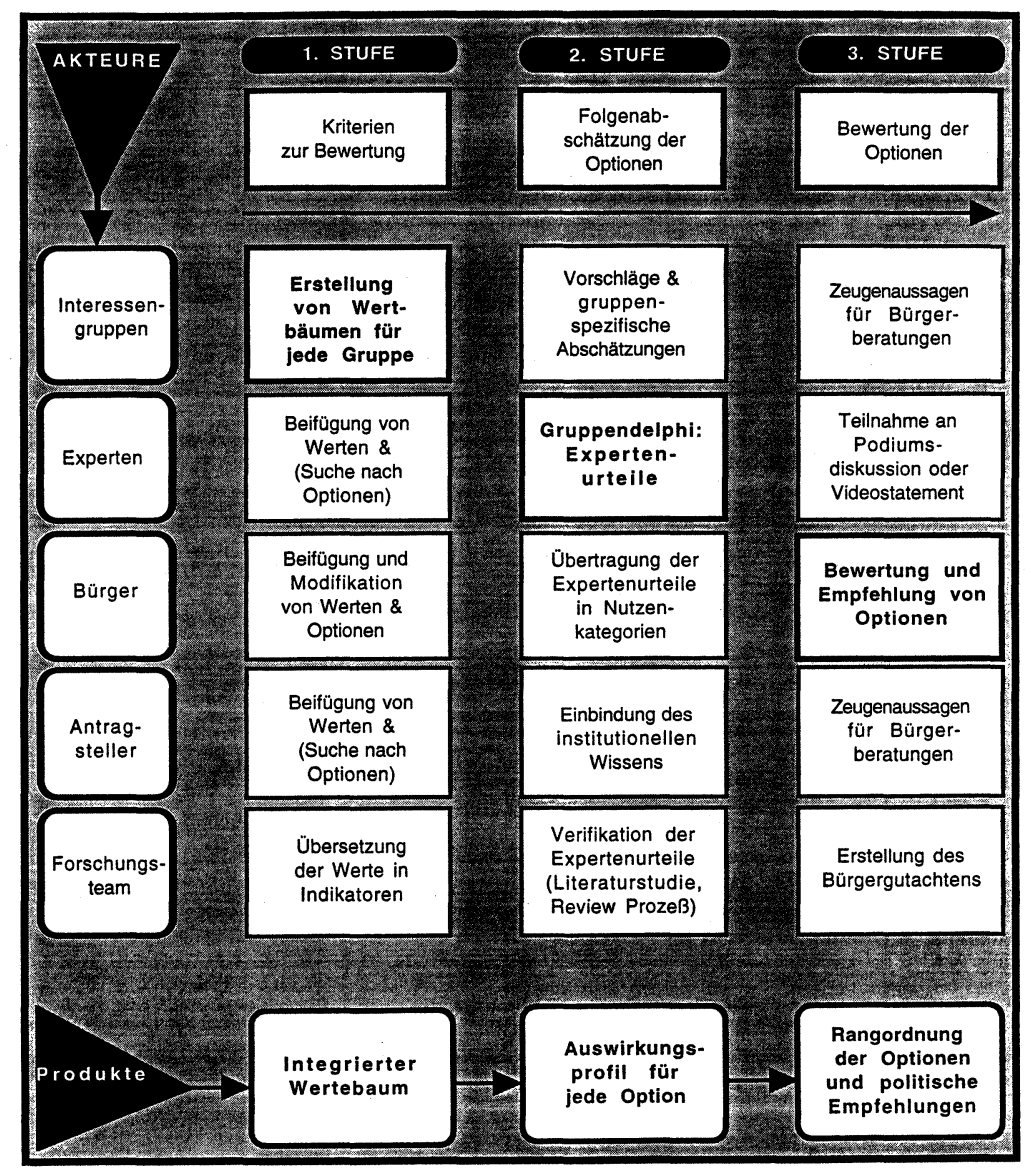

Neben der Strukturierung in drei Verfahrensschritte ist das Modell des kooperativen Diskurses durch eine verständigungsorientierte Vorgehensweise innerhalb der Beratungen der verschiedenen Gruppen (sei es Mediation, Delphi oder Bürgerforum) charakterisiert. Ziel des Diskurses ist ein Konsens über eine Handlungsempfehlung an die legalen Entscheidungsträger. Allerdings ist der Konsens nicht das einzig mögliche Ergebnis eines Diskurses. So kann es 
in Einzelfällen auch zu Kompromissen kommen, in anderen Fällen zu wohlbegründeten Dissensen oder Abstimmungsverfahren. Entscheidend ist auch bei den ,weicheren' Ergebnisformen, daß die Art der Entscheidungsfindung im Konsens bestimmt werden muß.

Das Verfahren des kooperativen Diskurses bietet den Vorteil, daß es zwischen Werterhebung, Faktenermittlung und Abwägung trennt und dafür verschiedene Verfahrensschritte vorschlägt. Darüber hinaus lassen sich die im dritten Schritt eingesetzten Bürgerforen beliebig vervielfachen. Mehrere parallel arbeitende Bürgerforen mit identischer Aufgabe bieten den besonderen Vorteil, daß zufällige gruppendynamische Entwicklungen oder Rückfälle in erfolgsorientierte Verhaltensweisen im Prinzip kompensiert werden können. Gleichzeitig wächst die legitimatorische Kraft der Empfehlungen, wenn mehrere unabhängig voneinander arbeitende Gruppen zu ähnlichen oder sogar identischen Empfehlungen kommen. ${ }^{9}$ Allerdings beruht der legitimatorische Effekt der Bürgerforen auf der expliziten Zusicherung der legalen Entscheidungsträger, die Empfehlung der Bürger zumindest zu berücksichtigen, wenn nicht sogar als verbindlich anzuerkennen. Das sequentielle Vorgehen und die Einbeziehung von Akteuren und Instrumenten ist in Bild 2 auf S. 191 illustriert.

\section{Fallbeispiel: Kooperativer Diskurs über eine Standortfestlegung ,Deponie Aargau Ost6}

\subsection{Vorgehensweise}

Das folgende Fallbeispiel beschreibt die Anwendung des kooperativen Diskurses für die Bestimmung einer Restabfalldeponie für den östlichen Teil des Kantons Aargau in der Schweiz (Webler 1994; Renn/Webler 1995; Renn u.a. 1996). Die Abfallbewirtschaftungsleitlinien des Kantons Aargau sehen vor, daß Reststoffe und Bauschutt auf Deponien abgelagert werden. Dabei sollte eine faire Lastenaufteilung auf die unterschiedlichen Regionen angestrebt werden. Während der westliche und nördliche Teil des Kantons bereits über eine Deponie verfügen bzw. eine solche in der Planung ist, stand der Bau einer Deponie im Süden des östlichen Kantonsteils noch aus. Aufgrund der zum Teil heftigen Proteste der Bevölkerung gegen die geplante Deponie in Suhr und wegen akuter Umweltprobleme mit zwei Deponie-Altlasten war das Baudepartement der Meinung, man müsse im Fall der Deponieplanung Aargau Ost die Bevölkerung umfassend und frühzeitig in das Planungsverfahren einbeziehen.

9 Entgegen aller intuitiver Vermutung ist dieser Fall relativ häufig. Die Erfahrungen mit Planungszellen als auch mit den von uns organisierten Bürgerforen hat gezeigt, daß die Urteile der Bürger in den parallelen Gruppen meist eng beieinander liegen. Gelegentlich kommen Ausreißer vor, die aber dann durch besondere Ereignisse erklärt werden können. 


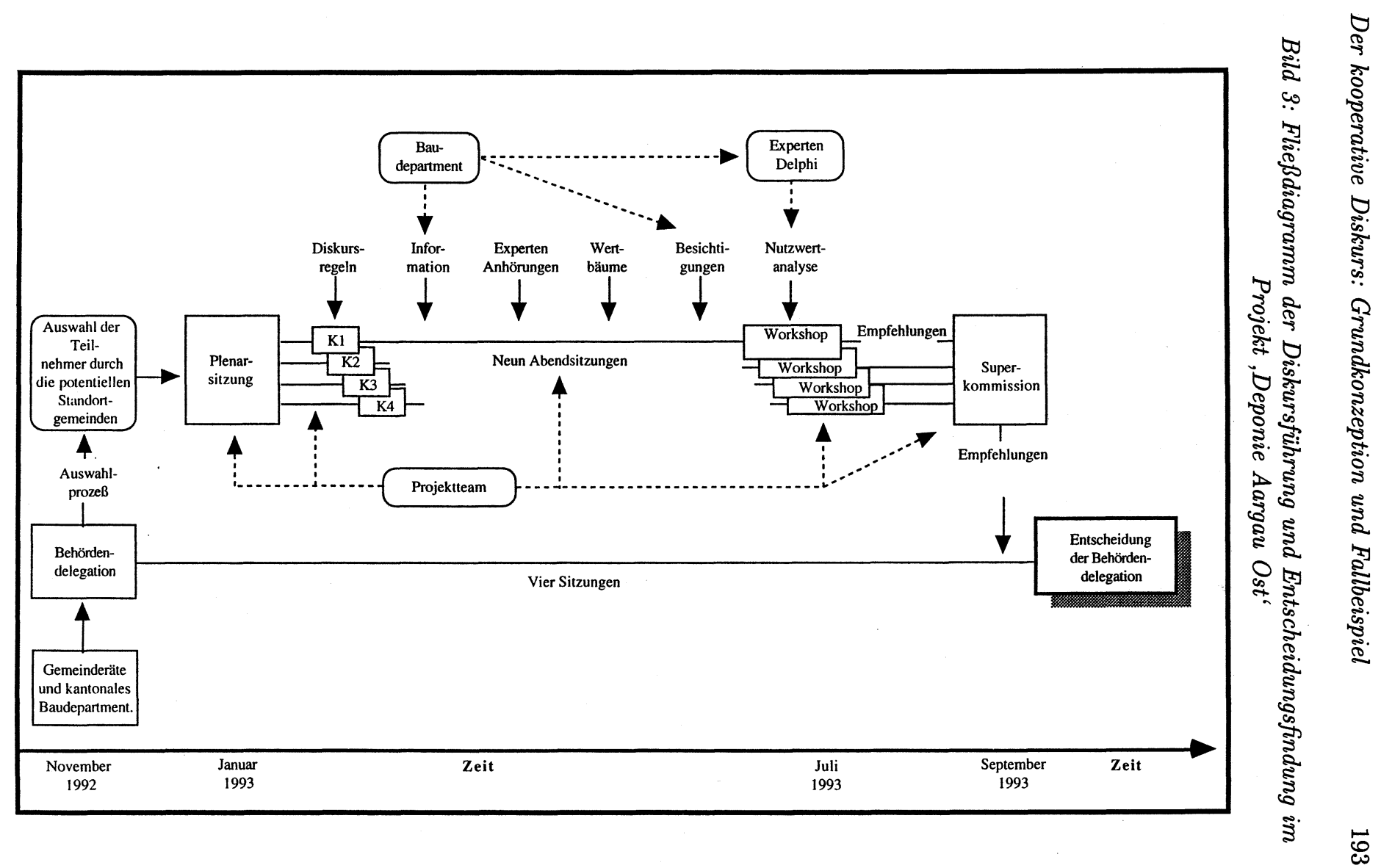


In einem ersten Schritt wurden 13 Gemeinden ausgewählt, die als mögliche Deponie-Standorte infrage kamen. Die weitere Auswahl von Standorten wurde dann durch einen kooperativen Diskurs mit Vertretern und Vertreterinnen aus den 13 Gemeinden vorgenommen. Das Baudepartement des Kantons beauftragte ein Forschungsteam der ETH Zürich unter unserer Leitung, das Diskursverfahren durchzuführen und zu begleiten. Ziel des Diskurses war es, aus den 13 Standorten eine Liste von zwei bis vier ernsthaften Kandidaten zu erstellen, die in der folgenden Erkundungsphase auf ihre Eignung überprüft werden sollten. Die Vorgehensweise ist in Bild 3 auf S. 193 illustriert. Aus jeder der 13 Gemeinden wurden acht Einwohner ausgewählt, von denen jeweils zwei in eine von vier Kommissionen berufen wurden. Somit waren in jeder Kommission gleich viele Repräsentanten aus jeder möglichen Standortgemeinde vertreten.

Die Aufgabe dieser Kommissionen bestand darin, Kriterien und Maßstäbe für die Bewertung der möglichen Standorte auszuarbeiten (Schritt 1 unseres Modells), die Standorte auf diesen Kriterien zu beurteilen und nach einer Gewichtung der Kriterien eine Rangordnung der möglichen Standorte festzulegen (Schritt 3). Zu diesem Zweck erhielten die Kommissionsmitglieder technische Informationen, sie konnten sich in Vorträgen und Anhörungen über Risiken und Probleme von Deponien informieren, sie besichtigten die potentiellen Standorte und führten eine systematische Bewertung dieser Standorte durch. Dazu erstellten sie zunächst einen hierarchisch geordneten Wertebaum, in dem alle Werte und Auswirkungsdimensionen, die für die Kommissionsmitglieder von Bedeutung waren, zusammengefaßt waren. Aus diesem Wertebaum wurden dann deduktiv Kriterien und Indikatoren abgeleitet.

Um die Beurteilung zu erleichtern, erhielten die Teilnehmer die Ergebnisse eines von uns im Frühjahr 1993 durchgeführten Experten-Workshops nach dem Delphi Verfahren (Schritt 2 unseres Modells). An diesem Gruppendelphi nahmen sieben Deponie-Experten teil, deren Aufgabe es war, für jeden Standort ein Eignungsprofil nach den von den Kommissionen erarbeiteten Kriterien zu erstellen. Dabei wurden nur die Kriterien bearbeitet, die Expertenwissen verlangten. Die Gewichtung der Kriterien blieb weiterhin den Kommissionsmitgliedern überlassen.

Zur abschießenden Bewertung der Standorte trafen sich alle vier Kommissionen zu getrennten Workshops, die ein bis zwei Tage dauerten. Ziel des Workshops war es, die Beurteilungen der Standorte und die Gewichtungen der Kriterien zusammenzufassen und in eine Art Nutzwertanalyse zu integrieren. Als Grundlage für die Beurteilungen dienten einerseits die Abschätzungen aus dem Experten-Delphi, andererseits die Resultate der geologischen Untersuchungen. Gleichzeitig spielten die persönlichen Eindrücke aus der Besichtigung der Standorte eine entscheidende Rolle. Die Bewertungen der Standorte auf den Kriterien wurden arbeitsteilig in kleinen Gruppen von fünf Personen 
innerhalb jeder Kommission vorbereitet und dann im Plenum diskutiert. Nach Verabschiedung der Bewertungen auf einer Skala von +2 bis -2 , rechnete der jeweilige Moderator die numerischen Nutzwerte aus und erstellte daraus eine Reihenfolge der Standorte.

Nach einer intensiven Diskussion der Nutzwertergebnisse wurde eine Reihenfolge der Standorte festgelegt. Zu Beginn der Kommissionssitzungen hatten sich die Teilnehmer auf ein mehrstufiges Entscheidungsverfahren im Konsens geeinigt. Angestrebt wurde zunächst eine konsensuale Lösung. Wenn diese nicht zustande kommen sollte, wurde ein eingeschränkter Konsens angestrebt, bei dem alle Kommissionsmitglieder der im Diskurs gefundenen Lösung zustimmen mußten, bis auf diejenigen, die direkt betroffen waren und die sich der Stimme enthalten konnten. Falls auch dieses Modell nicht zu realisieren sei, war eine qualifizierte Mehrheit von zwei Drittel bzw. drei Viertel der Stimmen erforderlich, um ein Gruppenvotum zu begründen. Nachdem alle Standortbewertungen durchgeführt worden waren, erwies sich die Argumentation für die jeweils gefundene Rangfolge als so einleuchtend, daß in allen vier Kommissionen einstimmige Ergebnisse erzielt wurden. Auch diejenigen, die als Anwohner von einem vorderen Platz in der Reihenfolge potentiell stärker betroffen waren, stimmten also dem jeweiligen Gruppenergebnis zu. Zusätzlich zu der Standortrangfolge erarbeiteten die Beteiligten auch Defizitausgleichstrategien für den Umgang mit nachteiligen Punkten der ansonsten als geeignet empfohlenen Standorte sowie allgemeine Empfehlungen für die Deponieplanung.

Von großem Interesse ist außerdem, daß sich die vier Kommissionen unabhängig voneinander für den gleichen Standort an der Spitze der Rangordnung entschieden. In der weiteren Rangfolge allerdings unterschieden sich die Empfehlungen der verschiedenen Kommissionen. Um diese Unterschiede auszugleichen, wurden aus den vier Kommissionen jeweils fünf Mitglieder ausgewählt, die in einem Kommissionsausschuß (Superkommission) die bestehenden Differenzen ausgleichen sollten. Im Herbst 1993 traf der Kommissionsausschuß zusammen und legte eine Reihenfolge der in Zukunft zu betrachtenden Standorte fest. Neben der von allen Kommissionen bestätigten ersten Priorität für den Standort Eriwies (bei Schinznach) empfahl die Superkommission an zweiter Stelle die möglichen Standorte Dietwil-Sins und Sins-Auw (gleichrangig) und an dritter Stelle die möglichen Standorte Abtwil und Uezwil. Diese Standorte sollen parallel auf ihre Eignung für eine Deponie weiter untersucht werden, wobei bei ähnlicher geologischer Eignung der jeweils ranghöhere Standort den Vorrang erhalten sollte.

Die Arbeit der Kommissionen wurde durch eine Behördendelegation begleitet, die sich aus jeweils einem Vertreter der potentiellen Standortgemeinden und dem Leiter des Baudepartements zusammensetzte. In unserem Modell des kooperativen Diskurses ist die Behördendelegation das Bindeglied zwischen den Kommissionen und der politischen Öffentlichkeit. Aus der Er- 
fahrung heraus, daß die Legitimationskraft der Empfehlungen weitgehend davon abhängt, daß sich die politischen Entscheidungsträger mit dem Verfahren und der ihm innewohnenden Logik identifizieren, hielten wir es für notwendig, die politischen Mandatsträger laufend zu informieren und gegebenenfalls auch um Rat zu bitten. Auf diese Weise wurde die Anschlußfähigkeit zwischen Bürgerkommissionen und offizieller Politik nicht nur über das einmalige Gutachten der Bürger, sondern auch über den Prozeß der laufenden Information und der Institutionalisierung eines ,Review Boards' in der Gestalt der Behördendelegation gewährleistet. Dementsprechend war es die Aufgabe der Behördendelegation, die Arbeiten der Kommissionen kritisch zu begleiten und deren Empfehlungen entgegenzunehmen, intensiv zu diskutieren und eine eigene Empfehlung für die weitere Bearbeitung der Deponiesuche zu formulieren. Im November 1993 schloß sich die Behördendelegation dem Votum des Kommissionsausschusses weitgehend an, wobei allerdings die Rangfolge der verbliebenen Standortmöglichkeiten nicht übernommen, sondern eine erneute Festlegung der Rangfolge nach weiteren geologischen Tests gefordert wurde.

\subsection{Evaluation des Verfahrens}

Alle Teilnehmer wurden zu Beginn der Kommissionsarbeit, kurz vor der Entscheidung über die Standortwahl und am Ende der Beteiligungsmaßnahme mit Hilfe eines formalisierten Fragebogens nach ihren Eindrücken, Erwartungen und Bewertungen befragt. Die Befragung der betroffenen Teilnehmer und Teilnehmerinnen sollte ein Bild des gesamten Verfahrens vermitteln und auch Veränderungen in der Einschätzung der Beteiligten sichtbar machen. Aus diesem Grund wurden insgesamt vier Befragungen durchgeführt. Die Resultate der Befragungen zeigen, daß die Erwartungen an den Diskurs hoch waren. Die Teilnehmer begannen ihre Kommissionsarbeiten mit einer positiven Grundeinstellung. Sie erwarteten von diesem Diskurs eine faire Mitbestimmung in einem Entscheidungsprozeß, der ihre Interessen betraf. $\mathrm{Da} ß$ in der dritten Befragung die Erfahrungen mit der gewählten Vorgehensweise mit den hohen Erwartungen übereinstimmten und in der vierten Befragung diese gar noch übertrafen, ist sicherlich als Erfolg für das Diskursverfahren zu bewerten.

Bei der subjektiven Beurteilung des Verfahrens wurde einzig die Effizienz der Vorgehensweise in allen drei Befragungen etwas weniger gut bewertet als die anderen Kategorien, was vielleicht ein Hinweis darauf ist, daß mit 8-10 Abendsitzungen und etlichen Samstagen für Besichtigungen und Workshops die Grenzen des freiwilligen Engagements erreicht sind und nach Möglichkeiten das Vorgehen weiter gestrafft werden müßte. Trotzdem schätzten die Beteiligten den Aufwand als dem Problem angemessen ein (Webler u.a. 1995).

Die Tatsache, daß die Beteiligten von Anfang an den Bau einer Deponie im östlichen Kantonsteil für notwendig hielten, bot eine gute Grundlage für 
den Diskurs. Das eingeschränkte Mandat einer Standortwahl war demnach bei allen Beteiligten akzeptiert. Trotzdem war das Problembewußtsein sowohl vor als auch nach den Kommissionsarbeiten ausgeprägt, schätzten doch die meisten Teilnehmer die geplante Deponie als langfristiges Risiko und als eine Gefährdung für die kommunale Lebensqualität ein. Allgemein veränderte sich die Einschätzung der Auswirkungen des geplanten Deponiebaus im Verlauf des Verfahrens kaum, obwohl in der vierten Befragung eine große Mehrheit angab, ihre Meinung bezüglich der Abfallproblematik aufgrund der Erfahrungen mit der Diskursarbeit geändert und neue Kenntnisse über Deponietechnik und mögliche Einwirkungen auf Mensch und Umwelt erworben zu haben.

Die Analyse der Beurteilungen durch die Gesamtheit der Kommissionsmitglieder zeigt eine weitgehende Zufriedenheit der Betroffenen mit der Art und Weise, wie sie in die Deponiestandortsuche eingebunden wurden. Die Teilnehmer waren der Überzeugung, daß das Verfahren seine primäre Aufgabe, nämlich Kriterien für eine Standortbewertung zu entwickeln und eine begründete Standortauswahl durchzuführen, erfüllt hat. Die Teilnehmer kritisierten die Zeitnot bei der Erarbeitung von Empfehlungen über mögliche Ausgleichsstrategien und die Vorgabe der Folgenanalyse durch die Experten des Gruppen-Delphi. Die Beteiligten äußerten sich dagegen zufrieden mit der Diskursleitung und gaben an, auf verschiedenen Gebieten der Abfallplanung und über das Abfallproblem neue Kenntnisse erworben zu haben.

In den Fällen in den USA und in Deutschland, in denen wir ähnliche Befragungen zu Beginn von Beteiligungsmaßnahmen haben durchführen lassen, erwiesen sich die Befragten als wesentlich skeptischer und mißtrauischer gegenüber dem Verfahren als im Fall Aargau (Renn u.a. 1985; 1989). Das mag damit zusammenhängen, daß wir in der Regel mit einer repräsentativen Stichprobe gearbeitet haben und nicht mit ausgewählten Kommissionsmitgliedern. Sicherlich spielt aber auch die politische Kultur der Schweiz mit der Betonung von freiwilliger Mitarbeit an politischen Entscheidungen (vor allem auf kommunaler Ebene) eine wichtige Rolle.

Seit 1993 führt die Akademie für Technikfolgenabschätzung in BadenWürttemberg in der Region Nordschwarzwald ein ähnliches Verfahren in einem noch größeren Maßstab durch. Nach einer ausführlichen Phase der Mediation zwischen organisierten Interessengruppen von 1994-1995 trafen sich von Januar bis Mai 1996 insgesamt 10 Bürgerforen (nach dem Zufallsverfahren ausgewählte Teilnehmer aus potentiell möglichen Standorten für Abfallbehandlungsanlagen), um über Standorte für biologisch-mechanische und thermische Anlagen zur Behandlung von Restabfall zu diskutieren. Ähnlich wie in der Schweiz wurden in allen 10 Foren einstimmige Ergebnisse erzielt, d.h. auch diejenigen Bürger stimmten den Empfehlungen zu, die ihren Heimatort als Standort vorsahen (Akademie 1996). Schwieriger war es dagegen, eine einvernehmliche Empfehlung aus den 10 einzelnen Gruppenvoten zu formu- 
lieren. In einer Art Superkommission (Delegiertenversammlung) mit jeweils drei Vertretern aus jedem Forum konnte zwar ein Kompromiß erzielt werden, der von allen Delegierten (wenn auch mit Zähneknirschen) akzeptiert wurde, dieser Kompromiß wurde aber nicht von allen 10 Gruppen bestätigt. An diesem Projekt wird die große Chance einer Konsensorientierung im Rahmen eines Diskurses deutlich, gleichzeitig erkennt man aber auch die Grenzen der Belastbarkeit solcher Konsense, sobald repräsentative Elemente, die eher mit erfolgsorientiertem Handeln in Beziehung stehen, mit ins Spiel kommen.

\subsection{Weitere Verfahrensweise mit den Diskursergebnissen im Aargau}

Nach Abschluß der Beratungen in den Kommissionen wurde das Baudepartement im Kanton Aargau beauftragt, alle verbleibenden Standorte parallel auf ihre Eignung zu überprüfen. Entgegen dem ursprünglichem Votum der Kommissionen wurde dabei die Rangfolge der noch als geeignet angesehenen Standorte nicht weiter in Betracht gezogen. Dies führte vor allem zu Irritationen im Ort Uezwil, der insgesamt bei der Beurteilung der Kommissionen im Mittelfeld landete, aber von vielen externen Experten bevorzugt wurde. Eine Reihe von Kommissionsmitgliedern sprach von Manipulation, obwohl die Behördendelegation ausdrücklich die Nennung einer Rangfolge so lange als verfrüht abgelehnt hatte, bis eindeutige geologische Ergebnisse vorliegen würden. Dieser Vorbehalt wurde aber von vielen als Freibrief für die Behörden verstanden, aus den insgesamt fünf verbleibenden Standorten denjenigen auszuwählen, der von Seiten der Behörden präferiert würde. Dieser Konflikt ist bis heute noch nicht ausgetragen. Da die Menge des Abfalls insgesamt auch in der Schweiz zurückgegangen ist, läßt man sich mit der Entscheidung eines weiteren Deponie-Standortes auch inzwischen mehr Zeit.

Im Anschluß an dieses Beteiligungsverfahren hat die Universität Zürich Befragungen von Bürgern aus den potentiellen Standorten durchgeführt, die selbst nicht an den Verhandlungen in den Kommissionen teilgenommen hatten. Dabei zeigten sich zwei interessante Ergebnisse. Zum einen konnten nicht einmal 30 Prozent der Befragten über das Beteiligungsverfahren Auskunft geben oder hatten davon gehört. Trotz starken Interesses der Medien und der politischen Öffentlichkeit lief das Verfahren offenkundig an den meisten Bürgern vorbei. Dies ist zwar erstaunlich, wenn man bedenkt, daß die Lebensqualität jedes Bürgers in den potentiellen Standortgemeinden unmittelbar berührt war, deckt sich aber andererseits mit den Ergebnissen vieler politikwissenschaftlicher Studien, nach denen politische Apathie aufgrund von Politikverdrossenheit und Pluralisierung von Lebensstilen stark zugenommen hat (Klages 1993; 49ff.; Gabriel 1994, 98ff.; Hepp 1994, 149ff.).

Das zweite interessante Ergebnis betraf die Bewertung des Verfahrens im 
Vergleich mit traditionellen Entscheidungsabläufen. Obgleich zwei Drittel der Befragten erst während der Umfrage von der Art des Beteiligungsverfahrens erfuhren und erst während der Befragung über die Vorgehensweise in knapper Form aufgeklärt wurden, also keinerlei Primärerfahrung hatten, wurde der diskursive Ansatz allen anderen Entscheidungsverfahren (Wahl durch Gemeinderat, Wahl durch Kanton, Wahl durch unabhängige Experten) mit Ausnahme des Referendums vorgezogen. Trotz geringer Kenntnis über die Vorgehensweise im Kanton Aargau war also der intuitive Legitimationseffekt relativ hoch. Ähnliche Erfahrungen wurden auch in Österreich bei der Standortbestimmung für eine Sondermüllanlage erzielt (Linnerooth-Bayer/Fitzgerald 1996). Dort wurde ebenfalls die diskursive Beteiligung betroffener Bürgern besser beurteilt als alle repräsentativen Entscheidungsverfahren. Interessanterweise wurde dort aber das unabhängige Expertengremium in der Rolle als unparteiischer Schiedsrichter noch besser beurteilt. Die technokratische Vorgehensweise fand offenbar mehr Zustimmung als das dezisionistische oder korporatistische Entscheidungsmodell.

\section{Diskussion der Ergebnisse und Zusammenfassung}

Versucht man eine Gesamtbilanz des Verfahrens im Aargau und ähnlicher Verfahren im In- und Ausland zu ziehen, dann scheinen sich im Aargau wie in vielen anderen Fällen die Erwartungen der Teilnehmer, der Antragsteller und der Genehmigungsbehörden weitgehend erfüllt zu haben (siehe auch Gaßner u.a. 1992, $77 \mathrm{ff}$.). Im konkreten Fall der Deponiesuche im Aargau sind an einigen Stellen Schwächen deutlich geworden: manche Informationen kamen zu spät, das Verfahren stand unter einem oft kontraproduktiven Zeitdruck, die technische Betreuung hätte verbessert werden können, und die Wahl des Kommissionsausschusses ließ zu wünschen übrig. Diese Schwächen konnten jedoch in einem kooperativen und konstruktiven Klima der gegenseitigen $\mathrm{Zu}-$ sammenarbeit weitgehend überwunden werden. In dieser Hinsicht gilt der Grundsatz, daß wohl kein Verfahren der Beteiligung so ideale Bedingungen schafft, daß böser Wille und Mangel an Kooperationsbereitschaft kompensiert werden könnten. Gleichzeitig helfen aber Kooperationsbereitschaft und der Wunsch zur konstruktiven Mitarbeit über manche strukturellen Schwächen der gewählten Vorgehensweise hinweg.

Wiewohl die Warnung vor übertriebenen Hoffnungen in Diskurse als Mittel der Umweltplanung und Konfliktlösung berechtigt ist (siehe etwa Knoepfel 1994, 84ff.), erscheint uns jedoch der Versuch, mit diskursiv strukturierten Verfahren der Bürgerbeteiligung zu arbeiten, nicht nur ein erforderlicher, sondern auch ein gangbarer Weg der Entscheidungsfindung zu sein. Die Notwendigkeit partizipativer Diskurse, gerade im Bereich der Umweltpolitik und Umweltplanung, ergibt sich aus der Problematik, daß kollektive Entscheidungen 
in immer stärkerem Maße zeitlich und räumlich weitreichende Konsequenzen haben, unser Wissen über diese Wirkungszusammenhänge immer komplexer und spezialisierter wird und die Bürger Mitspracherechte an der Gestaltung ihrer Lebenswelt einfordern (Renn/Oppermann 1995, 276). So sehr also diskursive Verfahren Umweltpolitik bereichern können und unter Umständen die in allen Umfragen zur Umweltpolitik zum Ausdruck kommende Politikverdrossenheit konstruktiv in Mitverantwortung überführen kann, so sind Diskurse aber keine magischen Instrumente zur Akzeptanzbeschaffung oder zur Systemintegration. Sie können ihr Ziel nur dann erreichen, wenn sie die vier Leitkriterien erfüllen: Fairneß, Kompetenz, Legitimation und Effizienz. Politisch wirksame und problemlösende Diskurse setzen Risikobereitschaft der politischen Entscheidungsträger, ein klares, aber offenes Mandat für die Diskursteilnehmer, eine ausreichende Ausstattung mit Ressourcen, Kompetenz in der Konzeption, Organisation und Führung von solchen Diskursen sowie eine klare Anbindung der Diskursergebnisse in den politischen Entscheidungsprozeß voraus. Solche Diskurse sind nicht zum Nulltarif zu haben.

Die moderne Gesellschaft ist darauf angewiesen, daß neue diskursive Formen in den Prozeß der politischen Urteils- und Entscheidungsfindung integriert werden müssen. Dazu bedarf es keiner grundlegenden Veränderungen der politischen Strukturen, sondern gezielter Reformen, die eine Anschlußfähigkeit der im Diskurs gewonnenen Ergebnisse in den Strukturrahmen der Politik sicherstellen. Gerade daran aber mangelt es heute noch. Das Fallbeispiel des Diskurses im Aargau zeigt, daß die Bewertungskriterien der Fairneß, der Kompetenz und der Effizienz (zumindest in Maßen) eingehalten worden sind. Natürlich wären auch hier weitere Verbesserungen möglich, aber von der theoretischen Konzeption wie von der praktischen Durchführung her erwiesen sich diese Qualitätskriterien als prinzipiell einlösbar. Das läßt sich für das Kriterium der Legitimität nur mit großen Einschränkungen behaupten. Zum einen ist es nicht gelungen, die politischen Entscheidungsträger mit dem Virus der verständigungsorientierten Vorgehensweise zu ,infizieren' oder zumindest die Anschlußfähigkeit zwischen Foren und Politik sicherzustellen. Die Institution der Behördendelegation war dabei sicherlich eine wichtige Hilfe, die jedoch im Anschluß an die eigentliche Beteiligungsmaßnahme an Bedeutung verlor, je mehr die Umsetzung der Ergebnisse zur politischen Routine wurde. Zum anderen war der Legitimationseffekt in der breiten Bevölkerung als eher gering einzustufen. Um in den Foren eine verständigungsorientierte Form der Argumentation zu gewährleisten, muß die Zahl der am runden Tisch versammelten Personen grundsätzlich beschränkt sein (Renn/Oppermann 1995, 272). Diese Beschränkung engt aber den Grad der kollektiven Verbindlichkeit, also den Legitimationsanspruch der erarbeiteten Empfehlungen ein. Deshalb sind diskursive Verfahren darauf angewiesen, zusätzliche Maßnahmen der Öffentlichkeitsarbeit und der Kommunikation mit außenstehenden Gruppen durch- 
zuführen. Dies ist bislang noch wenig gelungen. Auch das 1996 abgeschlossene Verfahren zur Planung der Restabfallbehandlung in der Region Nordschwarzwald, in dem massiv Öffentlichkeitsarbeit betrieben wurde, hatte mit dem Problem mangelnder öffentlicher Wahrnehmung zu kämpfen (Akademie 1996). Solange keine offenen Konflikte mit den entsprechenden öffentlichkeitswirksamen Ritualen zu vermelden waren, blieb das Interesse der Medien an Beteiligungsverfahren und Diskursen gering. Dementsprechend sind die Ergebnisse der Verfahren auch nur einem kleinen Kreis der Öffentlichkeit bekannt.

Legitimation bleibt also ein Problem bei der Beurteilung der Leistungsfähigkeit diskursiver Verfahren der Politikberatung und -vorbereitung. In der bisherigen Entwicklung von Beteiligungs- und Diskursverfahren sind bereits eine Reihe von Hürden genommen worden, die in der Vergangenheit häufig als unüberwindbar gegolten haben (siehe auch dazu den geschichtlichen Abriß der Beteiligung in Selle 1996). Kaum jemand hätte es noch vor einigen Jahren für möglich gehalten, daß in den meisten von uns betreuten Verfahren die von den Folgen der Entscheidungen betroffenen Bürger konsensuale Lösungen erarbeitet und gegen Druck von außen verteidigt haben. Wir sehen also die Probleme der Legitimation weniger als Ausdruck der prinzipiellen Unfähigkeit moderner Steuerungssysteme zur diskursiven Korrektur ihrer Handlungsroutinen, sondern vielmehr als eine Herausforderung für die Architekten und Organisatoren von solchen Diskursen, innovative Elemente des Verbindens zwischen Verständigung und Strategie, zwischen Argument und Aushandlung, zwischen Betroffenheit und Entscheidungshoheit, kurzum zwischen Sozialsystem und Politik zu entwickeln und zu erproben. Der hier vorgestellte kooperative Diskurs ist dabei eine der erfolgversprechenden Möglichkeiten, zu einer sinnvollen Konfliktaustragung und vorausschauenden Umweltplanung zu kommen. $\mathrm{Ob}$ solche Diskurse in Zukunft das politische System befruchten und bereichern können, wird maßgeblich davon abhängen, wie es gelingt, die Träger der Politik für Innovationen im politischen Entscheidungs- und Verwaltungshandeln zu gewinnen und die strukturellen Voraussetzungen zu schaffen, um verständigungsorientierte Empfehlungen zu einem festen Bestandteil der politischen Routinen zu machen. Erst dann kann der Diskurs als Element einer neuen politischen Kultur seine volle Geltungskraft entfalten.

\section{Bibliographie}

Akademie für Technikfolgenabschätzung (1994), Bürgergutachten. Bürgerbeteiligung an der Abfallplanung für die Region Nordschwarzwald. Bürgergutachten Teil I: Restabfallmengenprognose. Band 1: Empfehlungen. Band 2: Dokumentation, Stuttgart

- (1995), Bürgergutachten. Bürgerbeteiligung an der Abfallplanung für die Region Nordschwarzwald. Bürgergutachten Teil II: Technik der Restabfallbehandlung. Band 1: Empfehlungen, Stuttgart 
- (1996), Bürgergutachten, Bürgerbeteiligung an der Abfallplanung für die Region Nordschwarzwald. Bürgergutachten Teil III: Standortentscheidung durch Bürgerforen, Stuttgart

Akademie der Wissenschaften zu Berlin (1992), Umweltstandards, Berlin

Apel, K.-O. (1990), Diskursethik als politische Verantwortungsethik in der gegenwärtigen Weltsituation, in: B. Engholm/W. Röhrich (Hrsg.), Ethik und Politik heute. Verantwortliches Handeln in der technisch-industriellen Welt, Opladen, $37-55$

Bacow, L. S./M. Wheeler (1984), Environmental Dispute Resolution, New York

Beck, U. (1986), Die Risikogesellschaft. Auf dem Weg in eine andere Moderne, Frankfurt/Main

Biesecker, A. (1995), Kooperation, Netzwerk, Selbstorganisation - Prinzipien für eine faire und vorsorgende Ökonomie, in: A. Biesecker/K. Grenzdörffer (Hrsg.), Kooperation, Netzwerk, Selbstorganisation. Elemente demokratischen Wirtschaftens, Pfaffenweiler, 9-21

Brohm, W. (1990), Alternativen zum einseitigen hoheitlichenVerwaltungshandeln, in: Hoffmann-Riem/Schmidt-Aßmann (Hrsg.), 253-258

Bruder, W. (1980), Sozialwissenschaften und Politikberatung, Opladen

Burns, T.R./R. Überhorst (1988), Creative Democracy: Systematic Conflict Resolution and Policymaking in a World of High Science and Technology, New York

Chambers, S. (1992), Zur Politik des Diskurses: Risikieren wir unsere Rechte? in: K.-O. Apel/M. Kettner (Hrsg.), Zur Anwendung der Diskursethik in Politik, Recht und Wissenschaft, Frankfurt/Main, 168-186

Claus, F./P. M. Wiedemann (Hrsg.) (1994), Umweltkonflikte: Vermittlungsverfahren zu ihrer Lösung, Taunusstein

Consensus (1990), The Facility Siting ,Credo': Guidelines for Public Officials, in: Consensus 9, 5

Creighton, J. L. (1983), The Use of Values: Public Participation in the Planning Process, in: G. A. Daneke/M.W. Garcia/J. Delli Priscoli (eds.), Public Involvement and Social Impact Assessment, Boulder, 143-160

Crosby, N./J. M. Kelly/P. Schaefer (1986), Citizen Panels: A New Approach to Citizen Participation, in: Public Administration Review 46, 170-178

Cupps, D. S. (1977), Emerging Problems of Citizen Participation, in: Public Administraion Review 37, 478-487

Dahl, R. A. (1989), Democracy and its Critics, New Haven

DeSario, J./S. Langton (1987), Toward a Metapolicy for Social Planning, in: J. DeSario/S. Langton (eds.), Citizen Participation in Public Decision Making, Westport, 205-221

Dienel, P.C. (1978), Die Planungszelle, Opladen

- (1989), Contributing to Social Decision Methodology: Citizen Reports on Technological Projects, in: Vlek/Cvetkovich (eds.), 133-151

- /D. Garbe (1985), Zukünftige Energiepolitik. Ein Bürgergutachten, Edition, Technik und Sozialer Wandel ${ }^{\text {', München }}$

- /O. Renn, O. (1995), Planning Cells: A Gate to ,Fractal' Mediation, in: Renn/ Webler/Wiedemann (eds.), 117-140 
Döbert, R. (1992), Konsensustheorie als deutsche Ideologie, in: H.-J. Giegel (Hrsg.), Kommunikation und Konsens in modernen Gesellschaften, Frankfurt/Main, 276309

Dryzek, J. S. (1990), Discursive Democracy, Cambridge

Elster, J. (1989), Solomonic Judgments. Studies in the Limitation of Rationality, Cambridge

Etzioni, A. (1993), The Spirit of Community. Rights, Responsibilities and the Communitarian Agenda, New York

Fietkau, H.-J. (1994), Mediationsverfahren im Kreis Neuss, in: Claus/Wiedemann (Hrsg.), 99-118

Fiorino, D. (1990), Citizen Participation and Environmental Risk: A Survey of Institutional Mechanisms, in: Science, Technology, \& Human Values 15, 226-243

Folberg, J./A. Taylor (1984), Mediation: A Comprehensive Guide to Resolving Conflicts Without Litigation, San Francisco

Frey, B./F. Oberholzer-Gee (1996), Fair Siting Procedures: An Empirical Analysis of Their Importance and Characteristics, in: Policy Analysis and Management $15,353-376$

Führ, M. (1990), Mitbestimmungsmodell für Umweltentscheidungen? Stellungnahme des Öko-Instituts Darmstadt, Manuskript, Darmstadt

Gabriel, O. W. (1994), Politische Einstellungen und politische Kultur, in: O. W. Gabriel/F. Brettschneider (Hrsg.), Die EU-Staaten im Vergleich. Strukturen, Prozesse, Politikinhalte, Opladen, 96-133

Gaßner, H./L. M. Holznagel/U. Lahl (1992). Mediation. Verhandlungen als Mittel der Konsensfindung bei Umweltstreitigkeiten, Bonn

Greifenstein, R./P. Jansen/L. Kißler (1990), Partizipationskompetenz und technisch-organisatorische Innovation, in: L. Kißler (Hrsg.), Partizipation und Kompentenz. Beiträge aus der empirischen Forschung, Opladen, 15-54

Habermas, J. (1968), Technik und Wissenschaft als ,Ideologie', Frankfurt/Main

- (1971), Vorbereitende Bemerkungen zu einer Theorie der kommunikativen Kompetenz, in: J. Habermas/N. Luhmann (Hrsg.), Theorie der Gesellschaft oder Sozialtechnologie. Was leistet die Systemforschung?, Frankfurt/Main, 101-141

- (1981), Theorie des kommunikativen Handelns. Band 1 \& 2, Frankfurt/ Main

- (1991), Moralbewußtsein und kommunikatives Handeln, Frankfurt/Main

- (1992), Faktizität und Geltung. Beiträge zur Diskurstheorie des Rechts und des modernen Rechtsstaates, Frankfurt/Main

Hadden, S. (1995), Regulatory Negotiation as Citizen Participation: A Critique, in Renn/Webler/Wiedemann (eds.), 239-252

Hepp, G. (1994), Wertwandel und Bürgerbewußtsein heute, in: G. Hepp/S. Schiele/ U. Uffelmann (Hrsg.), Die schwierigen Bürger, Schwalbach/Taunus, 145-159

Hill, H. (1995), Mediation im Kontext neuerer Entwicklungen von Recht und Staat, in: Arbeitsgemeinschaft für Umweltfragen (Hrsg.), Umweltmediation in Deutschland. AGU aktuell Nr. 49, Bonn, 71-79

Himmelberger, J. J./S. J. Ratick/A. L. White (1991), Compensation for Risks: Host Community Benefits in Siting Locally Unwanted Facilities, in: Environmental Management 15, 647-658 
Hoffmann-Riem, W. (1990), Verhandlungslösungen und Mittlereinsatz im Bereich der Verwaltung: Eine vergleichende Einführung, in: Hoffmann-Riem/SchmidtAssmann (Hrsg.), 13-41

- /E. Schmidt-Assmann (Hrsg.) (1990), Konfliktbewältigung durch Verhandlungen. Bd I, Baden-Baden

Holznagel, B. (1986), Negotiation and Mediation: The Newest Approach to Hazardous Waste Facility Siting, in: Boston College Environmental Affairs Law Review 13, 329-378

- (1990), Konfliktlösung durch Verhandlungen, Baden-Baden

Jasanoff, S. (1982), Science and the Limits of Administrative Rule-Making: Lessons from the OSHA Cancer Policy, in: Osgoode Hall Law Journal 20, 536-561

Kathlene, L./J. Martin (1991), Enhancing Citizen Participation: Panel Designs, Perspectives, and Policy Formation, in: Policy Analysis and Management 10, 46-63

Keeney, R. L./O. Renn/D. von Winterfeldt (1987), Structuring West Germany's Energy Objectives, in: Energy Policy 15, 352-362

- / - / - /U. Kotte (1984), Die Wertbaumanalyse, Edition, Technik und Sozialer Wandel', München

Kelman, S. (1992), Adversary and Cooperationist Institutions for Conflict Resolution in Public Policymaking, in: Journal of Policy Analysis and Management 11, 178-206

Klages, H. (1993), Häutungen der Demokratie, Zürich

Knoepfel, P. (1994), Chancen und Grenzen des Kooperationsprinzips in der Umweltpolitik, in: OIKOS. Umweltökonomische Studenteniniative an der Hochschule St. Gallen (Hrsg.), Kooperationen für die Umwelt. Im Dialog zum Handeln, Zürich, 65-92

Kunreuther, H. (1995), Voluntary Siting of Noxious Facilities: The Role of Compensation, in: Renn/Webler/Wiedemann (eds.), 283-295

- /P. Portney (1991), Wheel of Fortune: A Lottery-Auction Mechanism for Siting of Noxious Facilities, in: Journal of Energy Engineering 117, 125-132

- /K. Fitzgerald/T. D. Aarts (1993), Siting Noxious Facilities: A Test of the Facility Siting Credo, in: Risk Analysis 13, 301-315

Linder, W./A. Vatter (1996), Kriterien zur Evaluation von Partizipationsverfahren, in: K. Selle (Hrsg.), Planung und Kommunikation, Wiesbaden-Berlin, 181-188

Linnerooth-Bayer, J./K. B. Fitzgerald (1996), Conflicting Views on Fair Siting Processes: Evidence from Austria and the U.S., in: Risk: Health, Safety $\&$ Environment 7, 119-134

Luhmann, N. (1984), Soziale Systeme: Grundriß einer allgemeinen Theorie, Frankfurt/Main

Mayntz, R. (1987), Politische Steuerung und gesellschaftliche Steuerungsprobleme. Anmerkungen zu einem theoretischen Paradigma, in: T. Ellwein/J. J. Hesse/R. Mayntz/F. W. Scharpf (Hrsg.), Jahrbuch zur Staats- und Verwaltungswissenschaft. Band 1, Baden-Baden, 89-100

O'Hare, M. (1977), Not on My Block You Don't: Facility Siting and the Strategic Importance of Compensation, in: Public Policy 25, 234-244 
- (1990), The Importance of Compensation and Joint Gains in Environmental Disputes, in: Hoffmann-Riem/Schmidt-Aßmann (Hrsg.), 191-204

- /L. Bacow/D. Sanderson (1983), Facility Siting and Public Opposition, New York

Parsons, T. (1967), Sociological Theory and Modern Society, New York

Prätorius, R. (1990), Streit, Konsens und politische Kultur: Vergleichende Perspektiven, in: U. Scarcinelli (Hrsg.), Demokratische Streitkultur. Theoretische Grundpositionen und Handlungsalternativen in Politikfeldern, Opladen, 63-76

Quinn, R. E./J. W. Rohrbaugh (1981), A Competing Values Approach to Organizational Analysis, in: Public Productivity Review 5, 141-159

Raiffa, H. (1982), The Art and Science of Negotiation, Cambridge

Renn, O. (1995), Style of Using Scientific Expertise: A Comparative Framework, in: Science and Public Policy No. 22, 147-156

- (1996), Möglichkeiten und Grenzen diskursiver Verfahren bei umwelrelevanten Planungen, in: A. Biesecker/K. Grenzdörfer (Hrsg.), Kooperation, Netzwerk, Selbstorganisation. Elemente demokratischen Wirtschaftens, Pfaffenweiler, 161197

- /G. Albrecht/U. Kotte/H. P. Peters/H. U. Stegelmann (1985), Sozialverträgliche Energiepolitik. Ein Gutachten für die Bundesregierung, Editon ,Technik und sozialer Wandel', München

- /R. Goble/D. Levine/H. Rakel/T. Webler (1989), Citizen Participation for Sludge Management. Final Report to the New Jersey Department of Environmental Protection, Clark University, Worcester

- /B. Oppermann (1995), Bottom-up' statt ,Top-down'. Die Forderung nach Bürgermitwirkung als (altes und neues) Mittel zur Lösung von Konflikten in der räumlichen Planung, in: Zeitschrift für Angewandte Umweltforschung. Sonderheft Stadtökologie, 257-276

- /T. Webler (1994), Konfliktbewältigung durch Kooperation in der Umweltpolitik. Theoretische Grundlagen und Handlungsvorschläge, in: OIKOS. Umweltökonomische Studenteninitiative an der Hochschule St. Gallen (Hrsg.), Kooperationen für die Umwelt. Im Dialog zum Handeln, Zürich, 11-52

- / - (1995), Der kooperative Diskurs: Theorie und praktische Erfahrungen mit einem Deponieprojekt im Aargau, in: P. Knoepfel (Hrsg.), Lösung von Umweltkonflikten durch Verhandlung. Beispiele aus dem In- und Ausland. Reihe Ökologie und Gesellschaft Band 10, Basel, 191-244

- / - /B. B. Johnson (1991), Public Participation in Hazard Management: The Use of Citizen Panels in the U.S., in: Risk: Issues in Health and Safety 2, 198-226

- / - /H. Kastenholz (1996), Procedural and Substantive Fairness in Landfill Siting: A Swiss Case Study, in: Risk: Health, Safety \& Environment 7, 145-168

- / - /H. Rakel/P. C. Dienel/B. Johnson (1993), Public Participation in Decision Making: A Three-Step-Procedure, in: Policy Sciences 26, 189-214

- / - /P. Wiedemann (eds.) (1995a), Competence and Fairness in Citizen Participation. Evaluating Models for Environmental Discourse, Dordrecht-Boston

- / - / - (1995b), The Pursuit of Fair and Competent Citizen Participation, in: Renn/Webler/Wiedemann (eds.), 339-368 
Rosenbaum, N. (1978), Citizen Participation and Democratic Theory, in: S. Langton (eds.), Citizen Participation in America, Lexington, 43-54

Rushefsky, M. (1984), Institutional Mechanisms for Resolving Risk Controversies, in: S. G. Hadden (eds.), Risk Analysis, Institutions, and Public Policy, Port Washington, 133-148

Saretzki, T. (1996), Wie unterscheiden sich Argumentieren und Verhandeln, in: V. von Prittwitz (Hrsg.), Verhandeln und Argumentieren. Dialog, Interessen und Macht in der Umweltpolitik, Opladen, 19-39

Scacinelli, U. (1990), Auf dem Weg in eine kommunikative Demokratie? Demokratische Streikultur als Element politischer Kultur, in: U. Scarcinelli (Hrsg.), Demokratische Streitkultur. Theoretische Grundpositionen und Handlungsalternativen in Politikfeldern, Opladen, 29-51

Selle, K. (1996), Von der Bürgerbeteiligung zur Kooperation und zurück, in: K. Selle (Hrsg.), Planung und Kommunikation, Wiesbaden-Berlin, 61-78

Shrader-Frechette, K. (1990), Scientific Method, Anti-Foundationalism, and Public Policy, in: Risk Issues in Health and Safety 1, 23-41

Striegnitz, M. (1990), Mediation: Lösung von Umweltkonflikten durch Vermittlung, in: Zeitschrift für Angewandte Umweltforschung 3, 51-62

Suhr, M. (1990), Die Bedeutung von Kompensationen und Entscheidungsverknüpfungen, in: Hoffmann-Riem/Schmidt-Aßmann (Hrsg.), 113-138

Sullivan, A. M. (1992), Siting Noxious Facilities: A Siting Lottery with Victim Compensation, in: Journal of Urban Economics 31, 360-374

Susskind, L. E./J. Cruishank (1987), Breaking the Impasse: Consensual Approaches to Resolving Public Disputes, New York

Vlek, C./G. Cvetkovich (eds.) (1989), Social Decision Methodology for Technological Projects, Dordrecht

Vollmer, H. (1996), Akzeptanzbeschaffung: Verfahren und Verhandlungen, in: Zeitschrift für Soziologie 25, 147-164

von Alemann, U./J. Heintze (1979), Neo-Korporatismus. Zur neuen Diskussion eines alten Begriffes, in: Zeitschrift für Parlamentsfragen 10, 469-482

von Schomberg, R. (1992), Argumentation im Kontext wissenschaftlicher Kontroversen, in: K.-O. Apel/M. Kettner (Hrsg.), Zur Anwendung der Diskursethik in Politik, Recht, Wissenschaft, Frankfurt/Main, 260-277

Webler, T. (1994), Experimenting with a New Democratic Instrument in Switzerland: Siting a Landfill in the Eastern Part of Canton Aargau. Arbeitsbericht im Rahmen des Polyprojekts: Risiko und Sicherheit technischer Systeme an der Eidgenössischen Technischen Hochschule, ETH Zürich

- (1995), ,Right' Discourse in Citizen Participation. An Evaluative Yardstick, in: Renn/Webler/Wiedemann (eds.), 35-86

- /H. Kastenholz/O. Renn (1995), Public Participation in Impact Assessment: A Social Learning Perspective, in: Environmental Impact Assessment Review 15, 443-463

- /D. Levine/H. Rakel/O. Renn (1991), The Group Delphi: A Novel Attempt at Reducing Uncertainty, in: Technological Forecasting and Social Change 39, 253263 
Wellmer, A. (1992), Konsens als Telos der sprachlichen Kommunikation? in: H.J. Giegel (Hrsg.), Kommunikation und Konsens in modernen Gesellschaften, Frankfurt/Main, 18-30

Wehrli-Schindler, B. (1987), Demokratische Mitwirkung in der Raumplanung, Bern

Wiedemann, P. M. (1994), Mediation bei umweltrelevanten Vorhaben: Entwicklungen, Aufgaben und Handlungsfelder, in: Claus/Wiedemann (Hrsg.), 177-194

- /F. Claus (1994), Konfliktmittlung bei umweltrelevanten Vorhaben. Ein Resümee, in: Claus/Wiedemann (Hrsg.), 228-235

Willke, H. (1995), Systemtheorie III. Steuerungstheorie, Stuttgart-Jena

Wissenschaftlicher Beirat der Bundesregierung (1996), Globale Umweltveränderungen, Welt im Wandel: Wege zur Lösung globaler Umweltprobleme. Jahresgutachten 1995, Berlin

Zilleßen, H. (1993), Die Modernisierung der Demokratie im Zeichen der Umweltpolitik, in: H. Zilleßen/P. C. Dienel/W. Strubelt (Hrsg.), Die Modernisierung der Demokratie, Opladen, 17-39 